\title{
Contrast independent localization of multiscale problems
}

\author{
Fredrik Hellman* Axel Målquist ${ }^{\dagger}$
}

November 21, 2018

\begin{abstract}
The accuracy of many multiscale methods based on localized computations suffers from high contrast coefficients since the localization error generally depends on the contrast. We study a class of methods based on the variational multiscale method, where the range and kernel of a quasiinterpolation operator defines the method. We present a novel interpolation operator for two-valued coefficients and prove that it yields contrast independent localization error under physically justified assumptions on the geometry of inclusions and channel structures in the coefficient. The idea developed in the paper can be transferred to more general operators and our numerical experiments show that the contrast independent localization property follows.
\end{abstract}

\section{Introduction}

High contrast and multiscale coefficients are frequently encountered in partial differential equations (PDEs) for a range of applications. Typical examples of such coefficients are the permeability field in porous media flow problems, varying by several orders of magnitude over short distances, and the rapidly varying heat conductivity in a composite material. In this paper, we focus on the multiscale method based on localized orthogonal decomposition (LOD) 18 and study how to improve its accuracy for high contrast coefficients. LOD is based on the framework of the variational multiscale method (VMS) [16 in the sense that the full solution space is decomposed into a coarse and a fine subspace, where this decomposition is determined by the range and kernel of a quasi-interpolation operator. A new low-dimensional multiscale space (subsequently used in a Galerkin or Petrov-Galerkin method) is constructed by computing coarse basis correctors in the fine space. The correctors have global support, but can be computed on localized patches around the support of the coarse basis functions. The approximability of the multiscale space is determined by the error introduced by the localization to patches, which in turn depends on the decay of the correctors within the patch. It was proven in [18, that this decay is exponential with respect to the radius of the patch, independent of the fine-scale variations of the coefficient but not generally independent of the contrast (ratio between largest and smallest value) of the coefficient. It has also been observed in numerical experiments that higher contrast coefficients lead to slower decay of the correctors, particularly within connected subdomains with large value of the coefficient, called channels. The decay of correctors (or the related concept of fine-scale Green's functions in VMS) in both one- and multi-dimensional settings was studied in [17] for different choices of projection operators and it was clearly shown that the choice of operator has a large impact on the decay rate.

In this paper, we study coefficients $A$ that take two values $\alpha$ and 1 , where $\alpha \ll 1$. This isolates the effect of high contrast while still capturing many interesting applications, such as composite materials and subsurface flows. We introduce a novel Clément-type quasi-interpolation operator $I_{H}$ (based on Scott-Zhang node variables) whose construction forces corrector decay within channels and prove that the localization error for this operator is independent of the contrast. The basic idea is to select the integration domain for each node variable in such a way the operator kernel admits a contrast independent Poincaré-type inequality within all channels and inclusions in the domain. In practice, this means that each connected channel and inclusion needs to have dedicated nodes placed along its extent with a

\footnotetext{
*Department of Information Technology, Uppsala University, Box 337, SE-751 05 Uppsala, Sweden. Supported by Centre for Interdisciplinary Mathematics (CIM), Uppsala University.

${ }^{\dagger}$ Department of Mathematical Sciences, Chalmers University of Technology and University of Gothenburg SE-412 96 Göteborg, Sweden. Supported by the Swedish Research Council.
} 
distance proportional to the mesh size of the coarse mesh. We present sufficient assumptions on the node placements and a proof for contrast free localization error when the decomposition is based on $I_{H}$. The properties of $I_{H}$ are studied both theoretically and numerically. We also present a related operator $I_{H, 1}$ which numerically performs even better, but for which our proof does not give any guarantees. They both, however, follow the basic idea of carefully selecting integration domain for the node variables, suggesting that this is a key to contrast independent localization.

In addition to the VMS based methods, the literature on numerical homogenization for elliptic multiscale problems includes the multiscale finite element method (MsFEM) [15, the generalized multiscale finite element method (GMsFEM) [8], the heterogeneous multiscale method (HMM) [7, and polyharmonic homogenization 21. The issue of high contrast coefficients without assumptions on periodicity has been addressed recently by many authors. For example, by using multiscale finite element approaches in [5, 9, flux norm approaches in [3, 20, and low-rank approximation of Green's functions in [2]. Our work has similarities to and has been inspired by the LOD based approach in [23], where contrast independent corrector decay results for $A$-weighted quasi-interpolation operators were shown under quasi-monotonicity (24]) assumptions on the coefficient distribution within the node patches. The idea of selecting integration domain for the node variables can be transferred also to the $A$-weighted quasi-interpolation operators, so that the integration domain is selected to guarantee quasi-monotonicity. We include numerical experiments for an $A$-weighted projective operator of that kind suggesting that carefully selecting integration domain is important for contrast independent localization.

The outline of the paper is as follows. Section 2 describes the model problem and gives a review of the localized orthogonal decomposition method for multiscale problems. Section 3 defines the interpolation operator $I_{H}$ and investigates its stability and approximability properties. Section 4 shows the contrast independent localization error following from using $I_{H}$ and presents the total error of the multiscale method. Section 5 briefly reviews a number of additional interpolation operators and presents a series of numerical experiments where the accuracy for methods based on the the different operators on high contrast problems is investigated. Finally, the numerical results are discussed and related to the theoretical findings.

\section{Problem formulation and numerical method}

As model problem, we consider the elliptic PDE, with a two-valued coefficient $A$,

$$
-\operatorname{div} A \nabla u=f
$$

on a polygonal domain $\Omega=\Omega^{1} \cup \Omega^{\alpha} \subset \mathbb{R}^{d}(d=1,2$ or 3$)$, where $\Omega^{1}$ and $\Omega^{\alpha}$ are disjoint. The coefficient attains two values $\left.A\right|_{\Omega^{1}}=1$ and $\left.A\right|_{\Omega^{\alpha}}=\alpha$ with $0<\alpha \leq 1$ in the two subdomains $\Omega^{1}$ and $\Omega^{\alpha}$. We impose homogeneous Dirichlet boundary conditions on $\Gamma \subset \partial \Omega$, and homogeneous Neumann boundary conditions on $\partial \Omega \backslash \Gamma$. We allow $\Gamma$ to be empty, in which case compatibility conditions $\int_{\Omega} u=0$ and $\int_{\Omega} f=0$ are imposed. We consider the case when $A$ is a high-contrast and highly oscillatory coefficient, i.e. that $\alpha \ll 1$, and that $\Omega^{1}$ and $\Omega^{\alpha}$ cannot be approximated well as a union of a set of elements, without having the element diameter be very small. Examples are given in Figure 1. Note that the coefficient is determined completely by $\Omega^{1}, \Omega^{\alpha}$ and $\alpha$.

We reformulate the problem on weak form. Let $V=\left\{v \in H^{1}(\Omega):\left.v\right|_{\Gamma}=0\right\}$ and $(\cdot, \cdot)$ denote the scalar product in $L^{2}(\Omega)$. We introduce a bilinear form $a$,

$$
a(u, v)=(A \nabla u, \nabla v),
$$

and assume $f \in L^{2}(\Omega)$. We restate our problem as to find $u \in V$,

$$
a(u, v)=(f, v)
$$

for all $v \in V$. With the bounds $\alpha \leq A \leq 1$, we have that $a$ is bounded and coercive and (2) admits a unique solution by the Lax-Milgram theorem.

We consider the following (coarse scale) finite element discretization. Let $\mathcal{T}_{H}$ be a family of conforming triangulations of $\Omega$ with mesh size parameter $H$. We denote the set of nodes by $\mathcal{N}_{H}$ and the set of free nodes by $\mathcal{N}_{H}^{\text {free }} \subset \mathcal{N}_{H}$. The elements are assumed to be shape regular and the meshes to be quasi-uniform, 


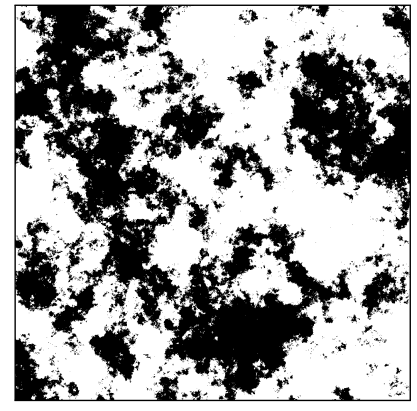

(a) Random field, resembling a heterogeneous porous medium.

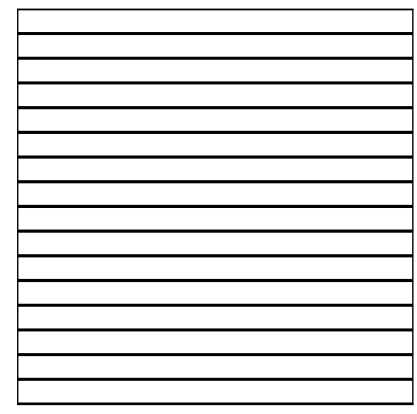

(b) Stripes, resembling cracks or composite materials.

Figure 1: Examples of coefficient $A$. The square is $\Omega$. Black color is $\Omega^{1}$ and white color is $\Omega^{\alpha}$.

i.e. we assume there is a constant $\rho$ independent of $H$ such that for all $H$,

$$
\max _{T \in \mathcal{T}_{H}} \frac{\operatorname{diam}(T)}{\operatorname{diam}\left(B_{T}\right)} \leq \rho \quad \text { and } \quad \max _{T, T^{\prime} \in \mathcal{T}_{H}} \frac{\operatorname{diam}(T)}{\operatorname{diam}\left(T^{\prime}\right)} \leq \rho,
$$

where $B_{T}$ denotes the largest ball contained in $T$. Let $S_{H}$ be the standard $\mathcal{P} 1 \mathrm{FE}$ space associated with $\mathcal{T}_{H}$. Further, let $V_{H}=S_{H} \cap V$, satisfying the Dirichlet boundary conditions. It is well known that convergence for the finite element method for this problem is generally not achieved unless the oscillations in $A$ are captured by the mesh. We briefly review the LOD method which allows for using low-dimensional spaces to find good approximations to problems with highly oscillatory coefficients. For more elaborate descriptions of different aspects of this method, see e.g. [11, 13, 18.

\subsection{Quasi-interpolation}

The first step of defining the LOD method is to choose a quasi-interpolation operator $\mathcal{I}_{H}$ from $V$ onto $V_{H}$. We call $V_{H}$ the coarse space and the infinte-dimensional $V$ the full space. The coarse space need not resolve the discontinuites of the coefficient $A$. In practice, the full space is typically also a finite-dimensional FE space, however, to better convey the new ideas in this work we use $V$ as full space, since it simplifies the exposition. See e.g. [18 for the fully discrete setting. The choice of a quasi-interpolation operator $\mathcal{I}_{H}: V \rightarrow V_{H}$ is crucial and defines a fine space $V^{\mathrm{f}}$ as its kernel, $V^{\mathrm{f}}=\operatorname{ker}\left(\mathcal{I}_{H}\right)=\left\{v \in V: \mathcal{I}_{H} v=0\right\}$.

Assumption A (Quasi-interpolation operator). We require the quasi-interpolation operator to satisfy:

1. $\mathcal{I}_{H}$ is a linear projection onto $V_{H}$,

2. there exists a constant $C_{\mathcal{I}}$ independent of $H$ such that for all $v \in V$ and all $T \in \mathcal{T}_{H}$, it holds

$$
H^{-1}\left\|v-\mathcal{I}_{H} v\right\|_{L^{2}(T)}+\left\|\nabla\left(v-\mathcal{I}_{H} v\right)\right\|_{L^{2}(T)} \leq C_{\mathcal{I}}\|\nabla v\|_{U(T)} .
$$

Here we define

$$
U(T)=\bigcup\left\{T^{\prime} \in \mathcal{T}_{H}: \bar{T} \cap \overline{T^{\prime}} \neq \emptyset\right\}
$$

i.e., the union of all neighboring elements to $T$.

There are many possible options for choosing $\mathcal{I}_{H}$ and consequently defining the fine space $V^{\mathrm{f}}$. We define the numerical method in an abstract setting where $\mathcal{I}_{H}$ only satisfies Assumption A and postpone to define a concrete instance of $\mathcal{I}_{H}$ to Sections 3 and 5.

\subsection{Localized orthogonal decomposition}

Given a fine space we define the non-local corrector operator $Q: V \rightarrow V^{\mathrm{f}}$ as an $A$-weighted Ritz-projection onto the fine space, find $Q v \in V^{\mathrm{f}}$, such that

$$
a\left(Q v, v^{\mathrm{f}}\right)=a\left(v, v^{\mathrm{f}}\right)
$$




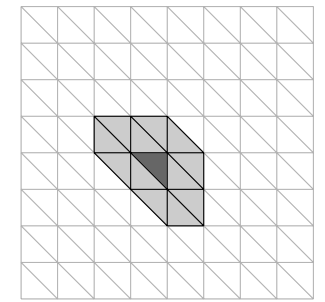

(a) One-layer element patch, $k=1$.

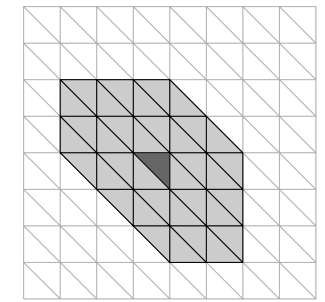

(b) Two-layer element patch, $k=2$.

Figure 2: Illustration of $k$-layer element patches. Dark gray is $T$. Light gray is $U_{k}(T)$.

for all $v^{\mathrm{f}} \in V^{\mathrm{f}}$. We introduce the multiscale space $V_{H}^{\mathrm{ms}}=(\operatorname{Id}-Q) V_{H}=\left\{v-Q v: v \in V_{H}\right\}$ and note that $\operatorname{dim}\left(V_{H}^{\mathrm{ms}}\right)=\operatorname{dim}\left(V_{H}\right)$. From (5) we observe that $V_{H}^{\mathrm{ms}}$ and $V^{\mathrm{f}}$ are orthogonal in the $a$-scalar product. Using the low-dimensional $V_{H}^{\mathrm{ms}}$ as test and trial space in a standard Galerkin method yields the following non-local multiscale method, find $u_{H}^{\mathrm{ms}} \in V_{H}^{\mathrm{ms}}$, such that

$$
a\left(u_{H}^{\mathrm{ms}}, v\right)=(f, v)
$$

for all $v \in V_{H}^{\mathrm{ms}}$. Galerkin orthogonality yields $a\left(u-u_{H}^{\mathrm{ms}}, v\right)=0$, i.e. the error $u^{\mathrm{f}}=u-u_{H}^{\mathrm{ms}}$ is in the fine space $V^{\mathrm{f}}$. Using that $\mathcal{I}_{H} v=0$ for $v \in V^{\mathrm{f}}$, we get an error bound (in energy norm, $\|\cdot\|^{2}=a(\cdot, \cdot)$ ), for the non-local multiscale method,

$$
\begin{aligned}
\left\|u^{\mathrm{f}}\right\|^{2} & =a\left(u^{\mathrm{f}}, u^{\mathrm{f}}\right)=\left(f, u^{\mathrm{f}}-\mathcal{I}_{H} u^{\mathrm{f}}\right) \\
& \leq\|f\|_{L^{2}(\Omega)}\left\|u^{\mathrm{f}}-\mathcal{I}_{H} u^{\mathrm{f}}\right\|_{L^{2}(\Omega)} \leq C_{\rho, \mathcal{I}} \alpha^{-1 / 2} H\|f\|_{L^{2}(\Omega)}\left\|u^{\mathrm{f}}\right\| .
\end{aligned}
$$

(Here, and in the remainder of the paper, $C$ denotes a function that depends on the variables or quantities listed in its subscript index list.) We note that this a priori error bound is independent of the solution regularity. In a practical implementation of this non-local multiscale method, the multiscale space $V_{H}^{\mathrm{ms}}$ is spanned by a basis $\left\{\phi_{i}-Q \phi_{i}\right\}_{i}$ where $\left\{\phi_{i}\right\}_{i}$ is the nodal basis of $V_{H}$. This requires the non-local corrector problem (5) to be solved for each basis function $\phi_{i}$ in the fine space, which is roughly as costly as solving the original problem for each basis function. However, in [18] it was proven that $Q \phi_{i}$ exhibits exponential decay from the support of $\phi_{i}$ and the corrector problems allow for localization with a small sacrifice in accuracy.

For the localization, we define patches $U_{k}(\omega) \subset \Omega$, where $0 \leq k \in \mathbb{N}$ and $\omega \subset \Omega$. With trivial case $U_{0}(\omega)=\omega, U_{k}(\omega)$ is defined by the recursive relation

$$
U_{k+1}(\omega)=\bigcup\left\{T^{\prime} \in \mathcal{T}_{H}: \overline{U_{k}(\omega)} \cap \overline{T^{\prime}} \neq \emptyset\right\} .
$$

We generalize the notation presented in Assumption A and let $U(\omega)=U_{1}(\omega)$. In particular, if $\omega=T \in$ $\mathcal{T}_{H}$, then $U_{k}(T)$ is a $k$-layer element patch around $T$. If $\omega=\{z\}$ and $z \in \mathcal{N}_{H}$, then (abusing notation) $U_{k}(z)$ is a $k$-layer node patch around $z$. See Figure 2 for an illustration of element patches.

Define localized fine spaces

$$
V^{\mathrm{f}}\left(U_{k}(T)\right)=\left\{v \in V^{\mathrm{f}}:\left.v\right|_{\Omega \backslash U_{k}(T)}=0\right\},
$$

consisting of fine functions which are zero outside patches. The localized corrector operator $Q_{k} v=$ $\sum_{T \in \mathcal{T}_{H}} Q_{k, T} v$ is a sum of localized patch corrector operators $Q_{k, T}: V \rightarrow V^{\mathrm{f}}\left(U_{k}(T)\right)$, defined by, find $Q_{k, T} \in V^{\mathrm{f}}\left(U_{k}(T)\right)$, such that

$$
a\left(Q_{k, T} v, v^{\mathrm{f}}\right)=\int_{T} A \nabla v \nabla v^{\mathrm{f}},
$$

for all $v^{\mathrm{f}} \in V^{\mathrm{f}}\left(U_{k}(T)\right)$. Note that the problem (8) is posed on the subdomain $U_{k}(T)$ only. This localization is what is behind the name localized orthogonal decomposition. In a practical setting, $Q_{k, T} \phi_{i}$ is solved for each coarse basis function $\phi_{i}$ and triangle $T$. Since $\phi_{i}$ has local support, the full localized corrector $Q_{k} \phi_{i}$ is a sum of only a few $Q_{k, T} \phi_{i}$. Now, a localized multiscale space $V_{H, k}^{\mathrm{ms}}=\left(\mathrm{Id}-Q_{k}\right) V_{H}$ is defined, 
spanned by a basis $\left\{\phi_{i}-Q_{k} \phi_{i}\right\}_{i}$. The corresponding localized multiscale method is, find $u_{H, k}^{\mathrm{ms}} \in V_{H, k}^{\mathrm{ms}}$, such that

for all $v \in V_{H, k}^{\mathrm{ms}}$.

$$
a\left(u_{H, k}^{\mathrm{ms}}, v\right)=(f, v)
$$

The localization introduces an error that depends on the patch size $k$. We can decompose the nonlocal multiscale solution $u_{H}^{\mathrm{ms}}=(\operatorname{Id}-Q) u_{H}$ for a unique $u_{H} \in V_{H}$. By Galerkin orthogonality using (2) and $(9)$, for any $v \in V_{H, k}^{\mathrm{ms}}$, in particular $v=\left(\operatorname{Id}-Q_{k}\right) u_{H}$, we have:

$$
\left\|u-u_{H, k}^{\mathrm{ms}}\right\|\|\leq\| u-v\|\|=\left\|u^{\mathrm{f}}\right\|\left|+\left\|\left(Q-Q_{k}\right) u_{H}\right\| \leq\|\| u^{\mathrm{f}}\left\|\mid+C_{\rho} \alpha^{-1} k^{d / 2} \theta(\alpha)^{k}\right\| f \|_{L^{2}(\Omega)},\right.
$$

where $0<\theta(\alpha)<1$ depends on the contrast $\alpha$ (see [18, Theorem 4.6] or [13, Theorem 3.7] for the last step.) The first term is the error from the non-local multiscale method (7), and the second term is the error from localization, which decays exponentially with patch size $k$. We note that for a given contrast, we can choose $k \approx \log H^{-1}$ to make the term $\left\|\left(Q-Q_{k}\right) u_{H}\right\|$ of the same order as $\left\|u^{\mathrm{f}}\right\| \|$, i.e. the error can be kept small by choosing appropriate patch sizes. However, we also note that the decay rate with respect to $k$ varies with $\alpha$. This is the contrast problem that we address in this paper. The effect of the contrast problem can be observed in the section of numerical experiments, Section 5. for quasi-interpolation operator $I_{H}^{\mathrm{SZ}}$.

\subsection{Right hand side correction}

Although the non-local multiscale method error $\left\|u^{\mathrm{f}}\right\|||$ converges with $H$ independent of the regularity of the solution $u$, we are interested in discarding this error to clearly distinguish the contrast dependency due to localization, i.e. how strongly $\alpha$ influences $\theta(\alpha)$ and the total error. This section describes how to compute a localized estimate $u_{k}^{\mathrm{f}}$ of $u^{\mathrm{f}}$ to the same accuracy as the localization.

Introduce the right hand side correction operator $R: V \rightarrow V^{\mathrm{f}}$,

$$
a\left(R v, v^{\mathrm{f}}\right)=\left(v, v^{\mathrm{f}}\right),
$$

for all $v^{\mathrm{f}} \in V^{\mathrm{f}}$. Then the error $u^{\mathrm{f}}=R f$. This problem can be split into a number of localized problems, one for each coarse triangle of which $f$ has support. We construct right hand side correction operators $R_{k, T}: V \rightarrow V^{\mathrm{f}}\left(U_{k}(T)\right)$, find $R_{k, T} f \in V^{\mathrm{f}}\left(U_{k}(T)\right)$ such that for all $v^{\mathrm{f}} \in V^{\mathrm{f}}\left(U_{k}(T)\right)$,

$$
a\left(R_{k, T} f, v^{\mathrm{f}}\right)=\int_{T} f v^{\mathrm{f}},
$$

in analogy with the localized corrector problems. Note that if $f$ has support only in a few triangles, only a few problems of the kind above needs to be solved. The full localized right hand side correction is $u_{k}^{\mathrm{f}}=R_{k} f=\sum_{T \in \mathcal{T}_{H}} R_{k, T} f$. It is again possible to show exponential decay of the localization error in terms of $k$,

$$
\left\|u^{\mathrm{f}}-u_{k}^{\mathrm{f}}\right\| \mid \leq C \alpha^{-1} k^{d / 2} \theta(\alpha)^{k}\left\|u^{\mathrm{f}}\right\| \| .
$$

Now, we have the following method (localized multiscale method with right hand side correction)

1. Compute $R_{k, T} f$ in (11) (localized patch problems) for all $T$ for which $\left.f\right|_{T} \neq 0$ and let $u_{k}^{\mathrm{f}}=$ $\sum_{T \in \mathcal{T}_{H}} R_{k, T} f$.

2. Compute all basis correctors $Q_{k, T} \phi_{i}$ by $(8)$ and let $\left\{\phi_{i}-Q_{k, T} \phi_{i}\right\}_{i}$ span $V_{H, k}^{\mathrm{ms}}$.

3. Find $u_{H, k}^{\mathrm{ms}, \mathrm{rhs}} \in V_{H, k}^{\mathrm{ms}}$, such that

$$
a\left(u_{H, k}^{\mathrm{ms}, \mathrm{rhs}}, v\right)=(f, v)-a\left(u_{k}^{\mathrm{f}}, v\right)
$$

for all $v \in V_{H, k}^{\mathrm{ms}}$.

4. The solution is $u_{H, k}=u_{H, k}^{\mathrm{ms}, \mathrm{rhs}}+u_{k}^{\mathrm{f}}$.

By Galerkin orthogonality using (2) and (12), for any $v \in V_{H, k}^{\mathrm{ms}}$, in particular $v=\left(\operatorname{Id}-Q_{k}\right) u_{H}$, we have:

$$
\begin{aligned}
\left\|u-u_{H, k}\right\| & \leq\left\|u-v-u_{k}^{\mathrm{f}}\right\|\left|\leq\left\|u^{\mathrm{f}}-u_{k}^{\mathrm{f}}\right\|\right|+\left\|\left(Q-Q_{k}\right) u_{H}\right\| \\
& \leq C \alpha^{-1} k^{d / 2} \theta(\alpha)^{k}\left(1+\alpha^{-1 / 2} H\right)\|f\|_{L^{2}},
\end{aligned}
$$

i.e. the factor $\theta(\alpha)$ influences all error terms, and the contrast dependency can be easily studied. 


\section{Geometry induced quasi-interpolation operator $I_{H}$}

This section defines a novel interpolation operator $I_{H}$. Several other operators are studied in the numerical experiments in Section 5 for evaluation on a series of high contrast coefficients. However, the theoretical results presented in this paper all concerns the operator $I_{H}$ defined in this section.

The main purpose of $I_{H}$ is to give rise to a space $V^{\mathrm{f}}$ in which correctors $Q v$ and right hand side corrections $R f$ decay fast with the distance from the support of $v$ and $f$, in order to give a small localization error. Numerical experiments show that the decay rate for classical Clément-type interpolation operators can be very low for high contrast coefficients. In the following, we give a heuristic argument to why the decay rate is low at high contrast. This argument also motivates the way $I_{H}$ is defined. We stress that the purpose of this argument is to illustrate the idea behind the construction of $I_{H}$, and it is not necessary for the results presented in this paper to hold.

Consider the corrector $Q v_{T}$ from (5) of a function $v_{T}$ that has support only in triangle $T$. For $d=1$, it is well-known (see e.g. [17]) that total element localization (i.e. $\left.Q v_{T}\right|_{\partial T}=0$ ) is possible by choosing the nodal interpolation operator to define $V^{\mathrm{f}}$. However, total element localization does not seem to be possible in higher dimensions. Thus, for the sake of this argument, we neglect the influence of $\mathcal{I}_{H}$ on $\left.Q v_{T}\right|_{\partial T}$ and set $\left.Q v_{T}\right|_{\partial T}=g$ to study the decay of $Q v_{T}$ outside $T$. Under this assumption, $Q v_{T}$ is the minimizer

$$
\left\|A^{1 / 2} \nabla Q v_{T}\right\|_{L^{2}(\Omega \backslash T)} \leq\left\|A^{1 / 2} \nabla v^{\mathrm{f}}\right\|_{L^{2}(\Omega \backslash T)} \quad \text { for all } v^{\mathrm{f}} \in V^{\mathrm{f}},
$$

with boundary conditions on $\partial \Omega$ and $\left.Q v_{T}\right|_{\partial T}=g$. We see from this minimization problem that derivatives $\left.\nabla Q v_{T}\right|_{\Omega^{1}}$ in $\Omega^{1}$ are more heavily penalized than derivatives $\left.\nabla Q v_{T}\right|_{\Omega^{\alpha}}$ in $\Omega^{\alpha}$, if the contrast is high. Thus, classical Clément-type interpolation operators $\mathcal{I}_{H}$ that use (possibly weighted) averages or local projections over full node patches as node variables, allows for the possibility to satisfy the requirement $\mathcal{I}_{H} Q v_{T}=0$ (i.e. $Q v_{T} \in V^{\mathrm{f}}$ ) by large variations of $\left.Q v_{T}\right|_{\Omega^{\alpha}}$, keeping $\left.Q v_{T}\right|_{\Omega^{1}}$ fairly constant (or slowly decaying to satisfy Dirichlet boundary conditions). This effect causes slow decay through channels where $A$ is large.

The idea behind the geometry induced operator $I_{H}$ is to dedicate a set of node variables (called class I nodes below) that do the averaging or local projections on subdomains of $\Omega^{1}$ only. Those subdomains are always connected subsets of $\Omega^{1}$ in order to admit a local Poincaré-type inequality. Class I nodes forces decay within $\Omega^{1}$ since large variations in $\Omega^{\alpha}$ cannot help to satisfy $I_{H} Q v_{T}=0$ if some node variables are defined in terms of values from $\Omega^{1}$ only. To construct $I_{H}$, there are certain conditions on the placement of nodes in relation to the coefficient. Although some of these conditions can be relaxed, the basic requirement (which is made precise below) is that all inclusions of $\Omega^{1}$ should contain nodes frequently enough. With enough nodes to dedicate at least one node to all connected inclusions, it is possible to construct $I_{H}$.

A similar idea is that of the $A$-weighted projective quasi-interpolation used in [23, in the sense that the projection integral of the latter operator (which is unconditionally taken over the full node patch) is weighted by $A$, so that variations within $\Omega^{\alpha}$ becomes less significant in the interpolation. However, instead of defining the operator in terms of the geometry of $\Omega^{1}$ within the node patch, there is a quasimonotonicity assumption of the coefficient distribution for contrast independent localization error.

The idea of selecting integration domain for the node variables is conveyed in this paper by considering a particular operator $I_{H}$ based on the Scott-Zhang-type node variable. This is, however, only a choice made in order to perform a concrete error analysis. The main idea can be applied also for other node variables. As an example of this, we will (without theoretical analysis) apply the idea also to the $A$ weighted projective quasi-interpolation operator in the numerical experiments.

\subsection{Scott-Zhang type node variables}

We briefly review Scott-Zhang type node variables [25]. We use $i$ and $j$ to index basis functions and denote by $\phi_{i}$ the nodal basis function of $S_{H}$ associated with node $z_{i}$. We introduce $N_{i}$ as a Scott-Zhangtype node variable corresponding to node $z_{i}$. To each node variable, we associate a domain $\sigma_{i} \subset \Omega$ that includes $z_{i}$. Based on this domain, we define an $L^{2}\left(\sigma_{i}\right)$-dual basis $\psi_{i}$, satisfying, for all basis functions $\phi_{j}$ of $S_{H}$,

$$
\int_{\sigma_{i}} \psi_{i} \phi_{j}=\delta_{i j}= \begin{cases}1 & i=j \\ 0 & i \neq j .\end{cases}
$$




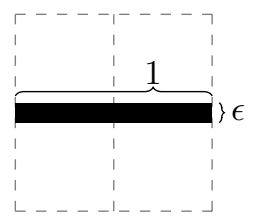

(a)

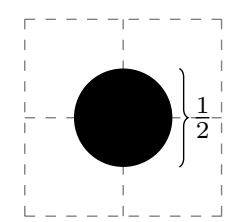

(b)

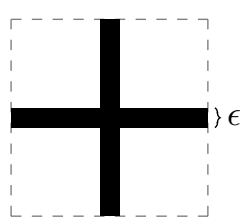

(c)

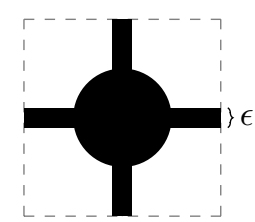

(d)

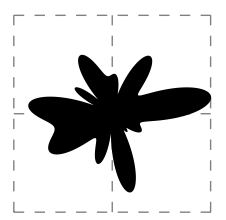

(e)

Figure 3: Five examples of shapes: (a) convex domain $p \leq \pi^{-1}$, (b) convex domain $p \leq(2 \pi)^{-1}$, (c) starshaped $p \leq \max \left(5 \pi^{-1}, \log \left(\epsilon^{-1}\right)^{1 / 2}\right)$, (d) chunky star-shaped $p \leq 5 \pi^{-1}$, (e) polar function star-shaped, point $(r, \varphi)$ in shape if $r \leq f(\varphi)$ with $\epsilon \leq f(\varphi) \leq 1$, then $p \leq \max \left(5 \pi^{-1}, \log \left(\epsilon^{-1}\right)^{1 / 2}\right)$.

This dual basis is used to define the node variable,

$$
N_{i}(v)=\int_{\sigma_{i}} \psi_{i} v
$$

\subsection{Geometry and mesh assumptions}

In order to define $I_{H}$, we impose the following assumptions on the geometry of $\Omega^{1}$ and the mesh. This is the formal condition for frequent enough node placement within $\Omega^{1}$.

Assumption B (Existence of a covering set of subdomains of $\Omega^{1}$ with diameter and Poincaré constants uniformly bounded by $H$ ). For a fixed $H$, there is a set $\left\{\omega_{1}^{1}, \ldots, \omega_{M}^{1}\right\}$ of $M$ open and possibly overlapping subdomains of $\Omega^{1}$, that satisfy the following conditions:

1. They cover exactly $\Omega^{1}$, i.e. $\overline{\bigcup_{i=1}^{M} \omega_{i}^{1}}=\overline{\Omega^{1}}$.

2. For each $\omega_{i}^{1}$, there is a free node $z_{i} \in \mathcal{N}_{H}^{\text {free }}$ such that $z_{i} \in \omega_{i}^{1}$.

3. There is a constant integer $\ell$ independent of $H$, such that $\omega_{i}^{1} \subset U_{\ell}\left(z_{i}\right)$.

4. There is a constant $p$ independent of $H$, such that the following Poincaré inequalities hold,

$$
\inf _{q \in \mathbb{R}}\|v-q\|_{L^{2}\left(\omega_{i}^{1}\right)} \leq p H\|\nabla v\|_{L^{2}\left(\omega_{i}^{1}\right)}, \quad i=1, \ldots, M .
$$

The constant $p$ carries information about the geometry of the subdomains. Non-chunky subdomains give rise to larger $p$. The constant $\ell$ relates the diameter of the subdomains to the mesh size (since we assume quasi-uniform meshes). Next, we illustrate by two examples the relation between geometry of $\Omega^{1}$ and the constants $p, \ell$ and the mesh.

Example 1 (The constants $p$ and $\ell$ ). In some applications, the subdomain $\Omega^{1}$ can be described as a union of similar shapes. Figure 3 gives five examples of possible shapes scaled to unit diameter and presents bounds on the corresponding Poincaré constants. The Poincaré constants have been generously estimated using the results for convex and star-shaped domains in [26, eq. (1.1), (1.2)]. For the examples shown, we note that the Poincaré constants for the shapes (a), (b) and (d) are bounded independently of the fine scale parameter $\epsilon$. The bound for shape (c) increases very slowly with $\epsilon$ and is reasonably bounded for most practical implementations.

These shapes can be translated and rotated without affecting their Poincaré constants. Scaling them by a factor $\ell H$ scales their Poincaré constants similarly. Thus, a union of these shapes (translated, rotated and scaled) can be used to construct subdomains $\Omega^{1}$ depicted in Figure 4

Example 2 (Mesh and subdomain matching). Consider the coefficient in Figure 4d and a mesh with mesh size $H$ with nodes in all circle centers, see Figure 5a We can decompose $\Omega^{1}$ into subdomains of the shape presented in Figure $3 \mathrm{~d}$ (after translation and $H$-scaling). One such subdomain is colored blue in Figure 5a. This choice of subdivision and mesh fulfills Assumption B.

Next, consider the refined mesh in Figure $5 \mathrm{~b}$. The decomposition of $\Omega^{1}$ into subdomains can now be done in a way yielding better Poincaré constants than for the previous mesh, using the shapes in Figure 3a and 3b. Three subdomains $\omega_{i}^{1}, \omega_{j}^{1}$, and $\omega_{k}^{1}$ are colored blue in the figure.

As the mesh is refined further, it is easy to see that it is possible to define subdomains satisfying the two assumptions so that their Poincaré constants can be bounded by $p$ and their diameter by $\ell H$ for some $p$ and $\ell$ independent of $H$. 


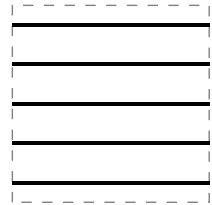

(a)

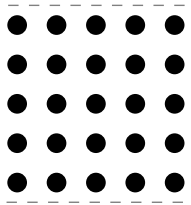

(b)

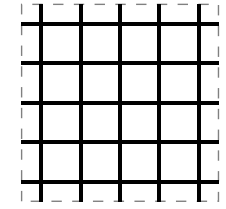

(c)

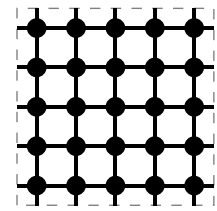

(d)

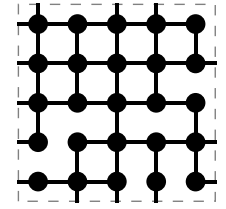

(e)

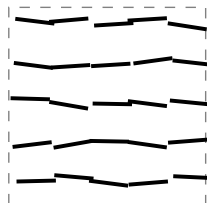

(f)

Figure 4: Six examples of coefficients. $\Omega^{1}$ is colored black and is a union of (possibly overlapping) subdomains like the ones presented in Figure 3 . (a-d) Periodic using a single shape, (e) non-periodic using several shapes, (f) non-periodic with randomly rotated and translated shapes.

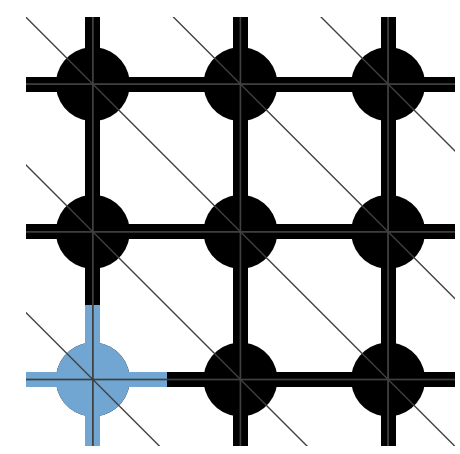

(a) Initial mesh.

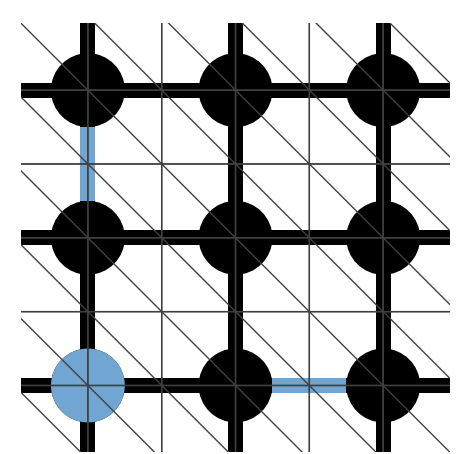

(b) One refinement.

Figure 5: Subdomain $\Omega^{1}$ is black and the triangular mesh is dark gray. In (a) one subdomain $\omega_{i}^{1}$ is blue and in (b) three subdomains $\omega_{i}^{1}, \omega_{k}^{1}$, and $\omega_{k}^{1}$ are blue.

\subsection{Definition of $I_{H}$}

The definition of $I_{H}$ is based on the Scott-Zhang node variables reviewed above, but with particular choices of integration domains $\sigma_{i}$. All free nodes are partitioned into two classes, and the domain is determined based on the class of the corresponding node. The class I nodes are dedicated to guarantee fast decay within $\Omega^{1}$ and are thus associated with the subdomains $\omega_{i}^{1}$ defined above.

I. The first class $\mathcal{N}_{H}^{\mathrm{I}} \subset \mathcal{N}_{H}^{\text {free }}$ is a selection of free nodes such that for each domain $\omega_{i}^{1}$ there is a $z_{i} \in \mathcal{N}_{H}^{\mathrm{I}}$ satisfying $z_{i} \in \omega_{i}^{1}$. We number these nodes $z_{i}$ for $i=1, \ldots, M$, although they are not necessarily unique.

II. The second class $\mathcal{N}_{H}^{\mathrm{II}}=\mathcal{N}_{H}^{\text {free }} \backslash \mathcal{N}_{H}^{\mathrm{I}}$ consists of all remaining free nodes.

Further, we define $\Sigma_{\delta}(z)=\{\delta(x-z)+z: x \in U(z)\}$, which is a node-centered $\delta$-scaling of the node patch of a node $z$, with $0<\delta \leq 1$. Now,

$$
I_{H, \delta} v=\sum_{i: z_{i} \in \mathcal{N}_{H}^{\mathrm{free}}} N_{i}(v) \phi_{i}
$$

where $N_{i}$ is defined in $(14)$, and

$$
\begin{array}{ll}
\sigma_{i}=U\left(z_{i}\right) \cap \omega_{i}^{1} & \text { for } z_{i} \in \mathcal{N}_{H}^{\mathrm{I}}, \\
\sigma_{i}=\Sigma_{\delta}\left(z_{i}\right) & \text { for } z_{i} \in \mathcal{N}_{H}^{\mathrm{II}} .
\end{array}
$$

The case $\delta=1 / 4$ defines $I_{H}=I_{H, \delta}$. See Figure 6 for an illustration of the two classes and their integration domain $\sigma_{i}$. Note that class I node variables integrate only over $\Omega^{1}$ since $\omega_{i}^{1} \subset \Omega^{1}$. The integration domain for class II nodes are restricted to the $\delta$-scaling of the node patch for technical reasons which are not fully understood, but appears necessary for the proof of contrast independent localization error (see Remark 1 below). An interesting special case is when $\delta=1$, i.e. that the full node patch is used also for class II nodes. Choosing $\delta=1$ defines the operator $I_{H, 1}$. We study $I_{H}$ both analytically and numerically and include $I_{H, 1}$ only in the numerical experiments for comparison. 


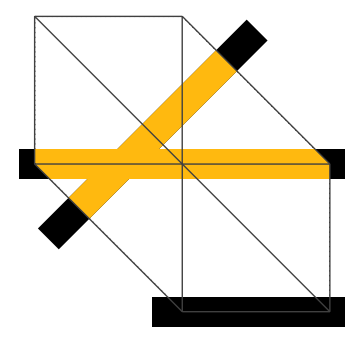

(a) Class I $\left(\mathcal{N}_{H}^{\mathrm{I}}\right)$.

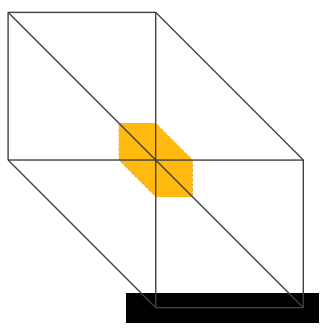

(b) Class II $\left(\mathcal{N}_{H}^{\mathrm{II}}\right)$.

Figure 6: Integration domain for two nodes of class I and II, respectively. Black is $\Omega^{1}$. Yellow is $\sigma_{i}$.

Remark 1. The theoretical results below holds for a slightly more general operator (which will not be discussed outside this remark) than $I_{H}$. Introduce a third class for nodes $z_{i}$ for which there are no channels or inclusions in its node patch, i.e. $U\left(z_{i}\right) \cap \Omega^{1}=\emptyset$. For those nodes, it is possible to choose integration domain as full node patch, $\sigma_{i}=U\left(z_{i}\right)$, and still have the contrast independent localization error results hold. That is, except for class I nodes, only nodes close to inclusions or channels actually need the integration domain restriction to the $\delta$-scaled node patch. As we will see in Section 5 , the numerical experiments for $I_{H, 1}$ even suggest that the restriction to $\delta$-scaled node patches is not necessary for any node.

\subsection{Node variable stability}

For class I nodes, the integration domains $\sigma_{i}$ are restricted to subsets of $\Omega^{1}$ and for class II nodes, they are restricted to the $\delta$-scaling of the node patch. The $L^{2}$-stability of the node variables $N_{i}$ is influenced by the corresponding $\sigma_{i}$. Thus, the stability and approximation properties of the full interpolation operator depends on the choice of $\delta$, the geometry of $\sigma_{i}$ and hence also on $\Omega^{1}$. In this subsection, we study the $L^{2}$-stability of $N_{i}(v)$ with respect to the integration domains $\sigma_{i}$. The results in this section hold also for general Scott-Zhang interpolation operators, i.e. for any choice $\sigma_{i} \subset U\left(z_{i}\right)$.

For the remainder of this section, we consider a single node variable $N_{i}$ and drop the index $i$ for all quantities associated with that node. Let $n$ be the number of basis functions with support in $\sigma$ and number those basis functions by $\phi_{j}, j=1, \ldots, n$. Without loss of generality, we assume $\phi_{1}(z)=1$. From the definition of $N$, we note that

$$
|N(v)| \leq\|\psi\|_{L^{2}(\sigma)}\|v\|_{L^{2}(\sigma)}
$$

and focus on $\|\psi\|_{L^{2}(\sigma)}$.

The next lemma shows how the (normalized) geometry of $\sigma$ affects the $L^{2}$-norm of the dual basis. An affine transform $F$ is used to rescale the physical node patch to a patch of diameter independent of $H$.

Lemma 2 (Dual basis $L^{2}$-norm). Let $F(\hat{x})=B \hat{x}+z$ for $B \in \mathbb{R}^{d \times d}$ with $\operatorname{det}(B)=H^{d}$, i.e. any affine transformation with volume rescaling proportional to the volume of $U(z)$. Define $\hat{\phi}_{j}(\hat{x})=\phi_{j}(F(\hat{x}))$ and $\hat{\sigma}=F^{-1}(\sigma)$. Let $\hat{M}_{j k}=\int_{\hat{\sigma}} \hat{\phi}_{k} \hat{\phi}_{j} \mathrm{~d} \hat{x}$, for $j, k=1, \ldots, n$ and $\hat{M}^{11}$ be the square submatrix of $\hat{M}$ where first row and column have been removed. Then, for the dual basis function $\psi$, we have

$$
\|\psi\|_{L^{2}(\sigma)}=H^{-d / 2} \kappa
$$

with $\kappa^{2}=\frac{\operatorname{det}\left(\hat{M}^{11}\right)}{\operatorname{det}(\hat{M})}$.

Proof. With the assumed node numbering, the definition of $\psi$ is

$$
\int_{\sigma} \psi \phi_{j}=\delta_{1 j}
$$

We can express $\psi=\sum_{k=1}^{n} \xi_{k} \phi_{k}$. Using the definition of $\psi$, we observe that $\|\psi\|_{L^{2}(\sigma)}^{2}=\int_{\sigma} \psi \sum_{j=1}^{n} \xi_{j} \phi_{j}=$ $\xi_{1}$. With $M_{j k}=\int_{\sigma} \phi_{k} \phi_{j} \mathrm{~d} x$ (and corresponding definition of $M^{11}$ ), the linear system of equations (17) 
can be expressed as $M \cdot \xi=(1,0, \ldots, 0)$, where $\xi=\left(\xi_{1}, \ldots, \xi_{d+1}\right)$. By Cramer's rule we get

$$
\xi_{1}=\frac{\operatorname{det}\left(M^{11}\right)}{\operatorname{det}(M)}=\operatorname{det}(B)^{-1} \frac{\operatorname{det}\left(\hat{M}^{11}\right)}{\operatorname{det}(\hat{M})} .
$$

The next lemma shows that the stability constant never increases by extending $\sigma$ within the node patch.

Lemma 3 (Extending integration domain never increases dual basis $L^{2}$-norm). If $\tilde{\sigma} \supset \sigma$, and $\tilde{\psi}$ is defined analogously to $\psi$, but with $\sigma$ replaced by $\tilde{\sigma}$ (and $n$ by $\tilde{n}$ ) then

$$
\|\tilde{\psi}\|_{L^{2}(\tilde{\sigma})} \leq\|\psi\|_{L^{2}(\sigma)} .
$$

Proof. Let $\psi=\sum_{k=1}^{n} \xi_{k} \phi_{k}$ and $\tilde{\psi}=\sum_{k=1}^{\tilde{n}} \tilde{\xi}_{k} \phi_{k}$ (note that $n \leq \tilde{n}$ ). Then we have that $\|\tilde{\psi}\|_{L^{2}(\tilde{\sigma})}^{2}=\tilde{\xi}_{1}$, and $\int_{\sigma} \tilde{\psi} \psi=\tilde{\xi}_{1}$. Using this, we get

$$
\|\tilde{\psi}\|_{L^{2}(\tilde{\sigma})}^{2}=\tilde{\xi}_{1}=\int_{\sigma} \tilde{\psi} \psi \leq\|\tilde{\psi}\|_{L^{2}(\sigma)}\|\psi\|_{L^{2}(\sigma)} \leq\|\tilde{\psi}\|_{L^{2}(\tilde{\sigma})}\|\psi\|_{L^{2}(\sigma)} .
$$

The stability constant $\kappa$ hence depends on the shape of $\hat{\sigma}$ in a node patch normalized coordinate system. We also note that $\kappa$ is a computable quantity. Next, we illustrate the relation between the shape of $\hat{\sigma}$ and $\kappa$ by studying a few examples in $2 \mathrm{D}$.

Example 3 (Constant $\kappa$ for four geometries of $\sigma$ when $d=2$ ). Consider the four geometries of $\sigma$ (restricted to a single element and normalized to unit element size) parametrized by $\epsilon$ depicted in Figure 7 . We are interested in the behavior of the stability constant $\kappa$ for small $\epsilon$. We compute elements of the matrix $\hat{M}$ up to high enough order of $\epsilon$ for it to be non-singular, and compute the lowest order term of $\kappa^{2}$ in terms of $\epsilon$.

(a) Here $\hat{M}=\frac{1}{24}\left(\begin{array}{lll}2 & 1 & 1 \\ 1 & 2 & 1 \\ 1 & 1 & 2\end{array}\right)$ and $\kappa^{2}=\operatorname{det}\left(\hat{M}^{11}\right) / \operatorname{det}(\hat{M})=18$.

(b) Here $\hat{M} \approx \frac{\epsilon}{12}\left(\begin{array}{ccc}4 & 2 & 3 \epsilon \\ 2 & 4 & 3 \epsilon \\ 3 \epsilon & 3 \epsilon & 4 \epsilon^{2}\end{array}\right)$ and $\kappa^{2}=\operatorname{det}\left(\hat{M}^{11}\right) / \operatorname{det}(\hat{M}) \approx 7 \epsilon^{-1}$ for $\epsilon \ll 1$.

(c) Here $\hat{M} \approx \frac{\epsilon^{2}}{24}\left(\begin{array}{ccc}12 & 4 \epsilon & 4 \epsilon \\ 4 \epsilon & 2 \epsilon^{2} & \epsilon^{2} \\ 4 \epsilon & \epsilon^{2} & 2 \epsilon^{2}\end{array}\right)$ and $\kappa^{2}=\operatorname{det}\left(\hat{M}^{11}\right) / \operatorname{det}(\hat{M})=18 \epsilon^{-2}$ for $0<\epsilon \leq 1$.

(d) Here we can reuse $\hat{M}$ from (b) (since (d) is a linear transformation of (b) with preserved volume) and compute $\kappa^{2}=\operatorname{det}\left(\hat{M}^{33}\right) / \operatorname{det}(\hat{M}) \approx 12 \epsilon^{-3}$ for $\epsilon \ll 1$.

(e) Here $\sigma$ is a superset of $\sigma$ in (c), thus according to Lemma $3, \kappa^{2} \leq 18 \epsilon^{-2}$.

Case (d) above can not occur with the presented definition of $I_{H}$, since $z \notin \sigma$. Although we do not elaborate on that, the condition $z \in \omega^{1}$ and consequently $z \in \sigma$ can be relaxed. However, it comes with the cost of larger $\kappa$.

Since, for class I nodes, the integration domain $\sigma$ is restricted to be subsets of a subdomain $\omega^{1}$, very narrow subomain $\omega^{1}$ can cause a large value of the stability constant $\kappa$ for the node it is paired with. For the remainder of this paper, we define an upper bound of the stability constants

$$
\kappa=\sup _{H} \max _{i: z_{i} \in \mathcal{N}_{H}} \kappa_{i}
$$




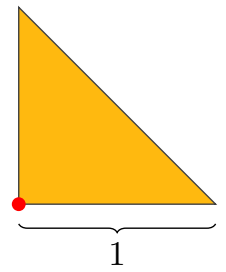

(a)

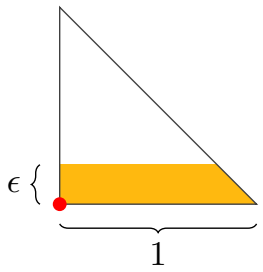

(b)

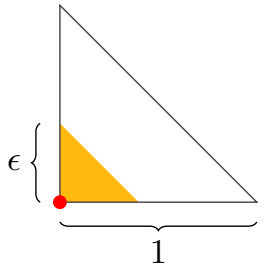

(c)

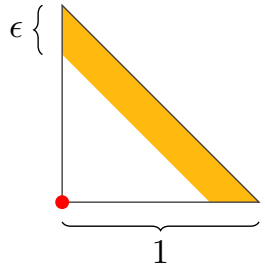

(d)

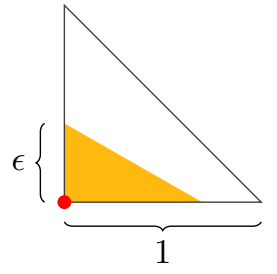

(e)

Figure 7: Red dot is the node $z$. Yellow area is the shape of the integration domain $\sigma$.

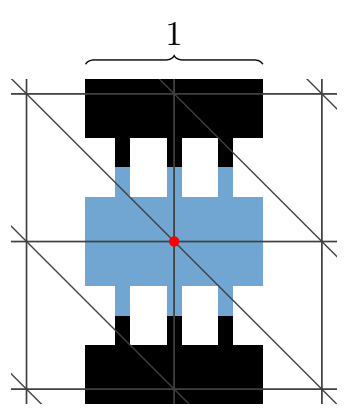

(a)

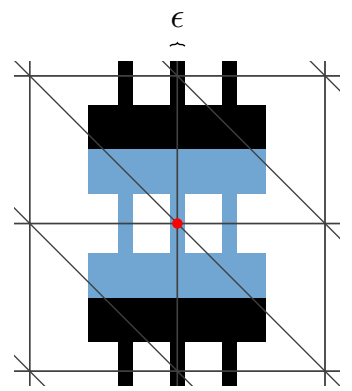

(b)

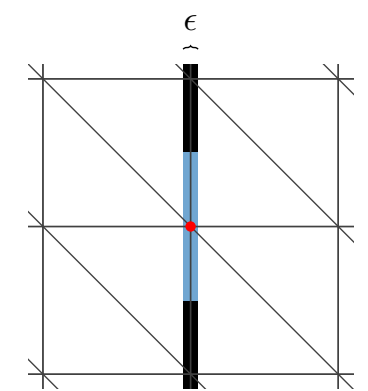

(c)

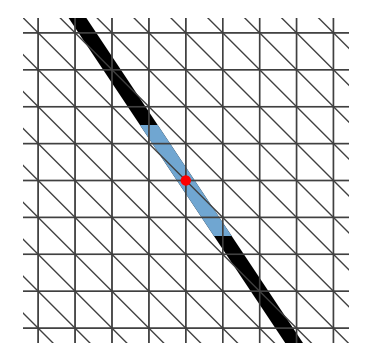

(d)

Figure 8: Illustrations for Example 4 . Black background is $\Omega^{1}$. Red dot is $z_{i}$. Blue area is $\omega_{i}^{1}$.

\subsection{Stability and approximability of $I_{H}$}

We present element stability and approximability results for $I_{H}$ based on the nodal variable stability above.

Lemma 4 (Stability and approximability of $I_{H}$ ). Under Assumptions $B$, for all $v \in V$, it holds that

$$
\begin{aligned}
\left\|v-I_{H} v\right\|_{L^{2}(T)} & \leq C_{\rho, \kappa} H\|\nabla v\|_{L^{2}(U(T))} & & \text { for all } T \in \mathcal{T}_{H}, \\
\left\|\nabla\left(v-I_{H} v\right)\right\|_{L^{2}(T)} & \leq C_{\rho, \kappa}\|\nabla v\|_{L^{2}(U(T))} & & \text { for all } T \in \mathcal{T}_{H},
\end{aligned}
$$

where $C_{\rho, \kappa}$ does not depend on $H$.

Proof. The proof follows closely the proofs in [25]. Let $D=\left\{i: z_{i} \in \bar{T}\right\}$ be the index set of the vertices in $T$.

$$
\begin{aligned}
\left|I_{H} v\right|_{H^{m}(T)} & =\left|\sum_{i \in D} N_{i}(v) \phi_{i}\right|_{H^{m}(T)} \leq C_{\rho} H^{-m+d / 2} \sum_{i \in D}\left|N_{i}(v)\right| \\
& \leq C_{\rho} H^{-m+d / 2} \sum_{i \in D}\left\|\psi_{i}\right\|_{L^{2}\left(\sigma_{i}\right)}\|v\|_{L^{2}\left(\sigma_{i}\right)} \stackrel{16}{\leq} C_{\rho} H^{-m} \max _{i \in D} \kappa_{i}\|v\|_{L^{2}(U(T))} .
\end{aligned}
$$

To prove (18), we use that for any $c \in \mathbb{R}$

$$
\begin{aligned}
\left\|v-I_{H} v\right\|_{L^{2}(T)} & \leq\|v-c\|_{L^{2}(T)}+\left\|I_{H}(v-c)\right\|_{L^{2}(T)} \\
& \leq\left(1+C_{\rho} \max _{i \in D} \kappa_{i}\right)\|v-c\|_{L^{2}(U(T))} \\
& \leq C_{\rho}\left(1+\max _{i \in D} \kappa_{i}\right) H\|\nabla v\|_{L^{2}(U(T))} .
\end{aligned}
$$

Bramble-Hilbert lemma was used in the last step for the element patch $U(T)$. A similar argument is used to prove 19.

The following example illustrates how node placements affects the stability constants $\ell, p$ and $\kappa$. 
Example 4 (The influence of node placement on stability constants). Consider the coefficients and node placement options $(\mathrm{a}-\mathrm{d})$ given in Figure 8 . We discuss the values of the constants based on the four options. We use [26, eq. (1.1), (1.2)] and [6, Remark 7.2] to estimate upper bounds of the Poincaré constant $p$, and the cases in Example 3 to estimate $\kappa$. We use the notation $A \lesssim B$ if $A \leq C B$ for a constant $C$ independent of $\epsilon$.

(a) Here $\omega_{i}^{1}$ is a union of three star-shaped domains with intersection of minimum width independent of $\epsilon$. Thus $p$ can be bounded from above independent of $\epsilon$. Node variable stability constant $\kappa$ is benign (and independent of $\epsilon$ ) since $\sigma_{i}$ covers a large and node centered area around the node. The subdomain is within the node patch, why $\ell=1$.

(b) Here $\omega_{i}^{1}$ is a union of three star-shaped domains with intersection of minimum width $\epsilon$, hence $p \lesssim$ $\epsilon^{-1} \log \left(\epsilon^{-1}\right)^{1 / 2}$, which increases when $\epsilon$ decreases. Node variable stability constant $\kappa$ is larger than case (a), but can be uniformly bounded independent of $\epsilon$. Again, $\ell=1$.

(c) Here $\omega_{i}^{1}$ is convex and $p \leq \pi^{-1}$, independent of $\epsilon$. Node variable stability is $\kappa \lesssim \epsilon^{-1 / 2}$ and $\ell=1$.

(d) Here the extent of $\omega_{i}^{1}$ is larger than the node patch, and $\ell=2$.

\section{Contrast independent error bounds for a multiscale method based on $I_{H}$}

This section presents error analysis of the localized methods based on $I_{H}$. Throughout this section, we fix $I_{H}$ as the choice of interpolation operator in the multiscale method and thus have $V^{\mathrm{f}}=\operatorname{ker}\left(I_{H}\right)$. The main result is that this yields error bounds where the exponential decay rate of the localization error is independent of the contrast $\alpha$.

The section is divided into three subsections. The first subsection presents a few bounds for functions in $V^{\mathrm{f}}=\operatorname{ker}\left(I_{H}\right)$. These constitute the key components for the proofs on contrast independent localization error, given in the second subsection. The error bounds for the full multiscale method are presented in the last and third subsection.

\subsection{Bounds for functions in $\operatorname{ker}\left(I_{H}\right)$}

We briefly discuss what is different between the contrast independent localization error proof in this paper and the contrast dependent classical proof in e.g. 18. The classical proof make use of inequalities of the kind

$$
\left\|A^{1 / 2} v^{\mathrm{f}}\right\|_{L^{2}(T)} \leq\left\|v^{\mathrm{f}}-I_{H} v^{\mathrm{f}}\right\|_{L^{2}(T)} \leq C \alpha^{-1 / 2} H\left\|A^{1 / 2} \nabla v^{\mathrm{f}}\right\|_{L^{2}(U(T))},
$$

where the contrast enters the bound. The results in this paper avoid this by using that $A$ is two-valued and splitting the integral separately over $T \cap \Omega^{1}$ and $T \cap \Omega^{\alpha}$. The node placement within $\Omega^{1}$ (guaranteed by Assumption $\mathrm{B}$ and the definition of $I_{H}$ yields a Poincaré-type inequality over $\Omega^{1}$ independent of $\alpha$ for functions in $\operatorname{ker}\left(I_{H}\right)$ as presented in (21) in Lemma 5 below. The norm on the right hand side in the estimate is taken over a larger domain than that on the left hand side. However, this spreading is restricted to $\Omega^{1}$, which keeps the separation of the initial integral to $\Omega^{1}$. For the integral over $\Omega^{\alpha}$, spreading is not a problem since we can carry the constant $\alpha^{1 / 2}$ to cancel the constant $\alpha^{-1 / 2}$ arising from the classical estimates.

Lemma 5 (Poincaré-type inequalities for functions in kernel to $I_{H}$ ). Under Assumptions $B$ and for all $v^{\mathrm{f}} \in V^{\mathrm{f}}=\operatorname{ker}\left(I_{H}\right)$, it holds that

$$
\begin{aligned}
\left\|v^{\mathrm{f}}\right\|_{L^{2}\left(\Omega^{1} \cap \omega\right)} & \leq C_{\rho, p, \kappa, \ell} H\left\|\nabla v^{\mathrm{f}}\right\|_{L^{2}\left(\Omega^{1} \cap U_{\ell}(\omega)\right)} & \text { for all } \omega \subset \Omega, \\
\left\|A^{1 / 2} v^{\mathrm{f}}\right\|_{L^{2}(T)} & \leq C_{\rho, p, \kappa, \ell} H\left\|A^{1 / 2} \nabla v^{\mathrm{f}}\right\|_{L^{2}\left(U_{\ell}(T)\right)} & \text { for all } T \in \mathcal{T}_{H},
\end{aligned}
$$

where $C_{\rho, p, \kappa, \ell}$ does not depend on $\alpha$ or $H$. 


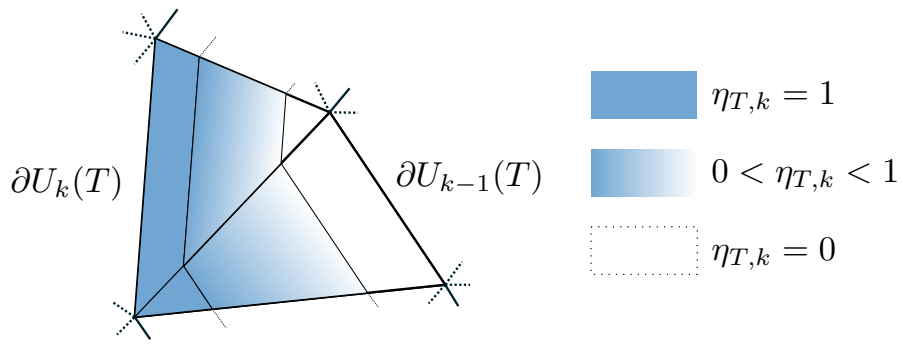

Figure 9: A 2D cut-off function illustrated.

Proof. For (21), we use that $N_{i}\left(v^{\mathrm{f}}\right)=0$ and $N_{i}(c)=c$, for any $c \in \mathbb{R}$. Then, using Assumption B and the node numbering $z_{i}$ described in Section 3.3 , choosing $c_{i} \in \mathbb{R}$ appropriately, we have for any $\omega \subset \Omega$,

$$
\begin{aligned}
\left\|v^{\mathrm{f}}\right\|_{L^{2}\left(\Omega^{1} \cap \omega\right)}^{2} & \leq \sum_{i: \omega_{i}^{1} \cap \omega \neq \emptyset}\left\|v^{\mathrm{f}}\right\|_{L^{2}\left(\omega_{i}^{1}\right)}^{2} \\
& \leq 2 \sum_{i: \omega_{i}^{1} \cap \omega \neq \emptyset}\left(\left\|v^{\mathrm{f}}-c_{i}\right\|_{L^{2}\left(\omega_{i}^{1}\right)}^{2}+\left\|N_{i}\left(v^{\mathrm{f}}-c_{i}\right)\right\|_{L^{2}\left(\omega_{i}^{1}\right)}^{2}\right) \\
& \leq 2 \sum_{i: \omega_{i}^{1} \cap \omega \neq \emptyset}\left(\left\|v^{\mathrm{f}}-c_{i}\right\|_{L^{2}\left(\omega_{i}^{1}\right)}^{2}+C_{\rho} \ell^{d} \kappa_{i}^{2}\left\|v^{\mathrm{f}}-c_{i}\right\|_{L^{2}\left(\sigma_{i}\right)}^{2}\right) \\
& \leq C_{\rho} \sum_{i: \omega_{i}^{1} \cap \omega \neq \emptyset}\left(1+\ell^{d} \kappa_{i}^{2}\right) p^{2} H^{2}\left\|\nabla v^{\mathrm{f}}\right\|_{L^{2}\left(\omega_{i}^{1}\right)}^{2} \\
& \leq C_{\rho, p, \kappa, \ell} H^{2}\left\|\nabla v^{\mathrm{f}}\right\|_{L^{2}\left(\Omega^{1} \cap U_{\ell}(\omega)\right)}^{2} .
\end{aligned}
$$

For $(22)$, we combine the two obtained results $(18)$ and $(21)$ to get

$$
\begin{aligned}
\left\|A^{1 / 2} v^{\mathrm{f}}\right\|_{L^{2}(T)} & \leq \alpha^{1 / 2}\left\|v^{\mathrm{f}}\right\|_{L^{2}\left(\Omega^{\alpha} \cap T\right)}+\left\|v^{\mathrm{f}}\right\|_{L^{2}\left(\Omega^{1} \cap T\right)} \\
& \leq \alpha^{1 / 2}\left\|v^{\mathrm{f}}-I_{H} v^{\mathrm{f}}\right\|_{L^{2}(T)}+C_{\rho, p, \kappa, \ell} H\left\|\nabla v^{\mathrm{f}}\right\|_{L^{2}\left(\Omega^{1} \cap U_{\ell}(T)\right)} \\
& \leq \alpha^{1 / 2} C_{\rho, \kappa} H\left\|\nabla v^{\mathrm{f}}\right\|_{L^{2}(U(T))}+C_{\rho, p, \kappa, \ell} H\left\|\nabla v^{\mathrm{f}}\right\|_{L^{2}\left(\Omega^{1} \cap U_{\ell}(T)\right)} \\
& \leq C_{\rho, p, \kappa, \ell} H\left\|A^{1 / 2} \nabla v^{\mathrm{f}}\right\|_{L^{2}\left(U_{\ell}(T)\right)}
\end{aligned}
$$

We define a slightly different cut-off function compared to previous works, which is constant on $\Sigma_{\delta}\left(z_{i}\right)$ for all nodes $z_{i}$. For the remainder of the paper we will set $\delta=1 / 4$ as in the definition of $I_{H}$ and drop the $\delta$-subscript. The operator $I_{H, 1}$ for which $\delta=1$ will not be analyzed and appears again in the numerical experiment section. We introduce, for $T \in \mathcal{T}_{H}$ and $k>0$, the auxiliary function $\hat{\eta}_{T, k} \in S_{H}$,

$$
\begin{array}{ll}
\hat{\eta}_{T, k}(x)=0 & \text { for } x \in U_{k-1}(T), \\
\hat{\eta}_{T, k}(x)=1 & \text { for } x \in \Omega \backslash U_{k}(T),
\end{array}
$$

and define the cut-off function as $\eta_{T, k}=\max \left(0, \min \left(1,2 \hat{\eta}_{T, k}-1 / 2\right)\right)$. We get $\left\|\nabla \eta_{T, k}\right\|_{L^{\infty}(\Omega)} \leq 2 C_{\rho} H^{-1}$. Figure 9 shows an illustration of such a cut-off function.

With the definition of the cut-off functions at hand, we can study a second term which appears in the classical proof and where contrast enters the bound, namely

$$
\left\|A^{1 / 2} \nabla I_{H}\left(\eta v^{\mathrm{f}}\right)\right\|_{L^{2}(T)} \leq C\left\|\nabla\left(\eta v^{\mathrm{f}}\right)\right\|_{L^{2}(U(T))} \leq C \alpha^{-1 / 2}\left\|A^{1 / 2} \nabla v^{\mathrm{f}}\right\|_{L^{2}\left(U_{2}(T)\right)},
$$

for a cut-off function $\eta$. With $I_{H}$, this term can also bounded independently of $\alpha$ using similar techniques as in the previous lemma. 
Lemma 6 (Local contrast independent energy norm stability of fine space functions after cut-off and interpolation). If $\eta \in L^{\infty}(\Omega)$ is constant in $\Sigma\left(z_{i}\right)$ for all nodes $z_{i}$ and $\|\eta\|_{L^{\infty}(\Omega)} \leq 1$, then for any $v^{\mathrm{f}} \in V^{\mathrm{f}}$ and $\omega \subset \Omega$,

$$
\left\|A^{1 / 2} \nabla\left(I_{H}\left(\eta v^{\mathrm{f}}\right)\right)\right\|_{L^{2}(\omega)} \leq C_{\rho, p, \kappa, \ell}\left\|A^{1 / 2} \nabla v^{\mathrm{f}}\right\|_{L^{2}\left(U_{\ell+2}(\omega)\right)},
$$

where $C_{\rho, p, \kappa, \ell}$ does not depend on $\alpha$ or $H$.

Proof. We split the integral into one contribution over $\Omega^{1}$ and one over $\Omega^{\alpha}$. For $\Omega^{1}$,

$$
\begin{aligned}
\left\|\nabla\left(I_{H}\left(\eta v^{\mathrm{f}}\right)\right)\right\|_{L^{2}\left(\Omega^{1} \cap \omega\right)}^{2} & =\int_{\Omega^{1} \cap \omega}\left(\sum_{i: z_{i} \in \mathcal{N}_{H}} N_{i}\left(\eta v^{\mathrm{f}}\right) \nabla \phi_{i}\right)^{2} \\
& \leq C_{\rho} \sum_{i: z_{i} \in \mathcal{N}_{H}}\left(\int_{\sigma_{i}} \psi_{i} \eta v^{\mathrm{f}} \cdot \int_{\Omega^{1} \cap \omega} \nabla \phi_{i}\right)^{2} \cdots
\end{aligned}
$$

We study the two integrals. The following step justifies the partition into the two classes introduced in Section 3.3 For any node $z_{i} \in \mathcal{N}_{H}^{\mathrm{II}}, \eta$ is constant in $\sigma_{i}$ and $\int_{\sigma_{i}} \psi_{i} \eta v^{\mathrm{f}}=\eta \int_{\sigma_{i}} \psi_{i} v^{\mathrm{f}}=0$ since $v^{\mathrm{f}} \in V^{\mathrm{f}}$. This makes the corresponding term in the sum is zero. We define the set $\mathcal{N}_{\omega}^{1}=\left\{z_{i}: z_{i} \in \mathcal{N}_{H}^{\mathrm{I}}\right.$ and $U\left(z_{i}\right) \cap$ $\Omega^{1} \cap \omega \neq \emptyset$, which contains all nodes whose corresponding term in the sum is not zero in general, and continue using mesh quasi-uniformity, Lemma 2 , that $\|\eta\|_{L^{\infty}(\Omega)} \leq 1$, and $\sigma_{i} \subset U\left(z_{i}\right) \cap \Omega^{1}$ for $z_{i} \in \mathcal{N}_{\omega}^{1}$,

$$
\begin{aligned}
\cdots & \leq C_{\rho} H^{-2-d} \sum_{i: z_{i} \in \mathcal{N}_{\omega}^{1}}\left(\int_{\sigma_{i}} \psi_{i} \eta v^{\mathrm{f}}\right)^{2} \\
& \stackrel{16]}{\leq} C_{\rho, \kappa} H^{-2} \sum_{i: z_{i} \in \mathcal{N}_{\omega}^{1}}\left\|v^{\mathrm{f}}\right\|_{L^{2}\left(\sigma_{i}\right)}^{2} \\
& \leq C_{\rho, \kappa} H^{-2} \sum_{i: z_{i} \in \mathcal{N}_{\omega}^{1}}\left\|v^{\mathrm{f}}\right\|_{L^{2}\left(\Omega^{1} \cap U\left(z_{i}\right)\right)}^{2} \\
& \quad \leq C_{\rho, p, \kappa, \ell} \sum_{i: z_{i} \in \mathcal{N}_{\omega}^{1}}\left\|\nabla v^{\mathrm{f}}\right\|_{L^{2}\left(\Omega^{1} \cap U_{\ell+1}\left(z_{i}\right)\right)}^{21} \\
& \leq C_{\rho, p, \kappa, \ell}\left\|\nabla v^{\mathrm{f}}\right\|_{L^{2}\left(\Omega^{1} \cap U_{\ell+2}(\omega)\right)}^{2}
\end{aligned}
$$

where we used shape regularity and that $\bigcup_{i: z_{i} \in \mathcal{N}_{\omega}^{1}} U_{\ell+1}\left(z_{i}\right) \subset U_{\ell+2}(\omega)$ in the last step. For $\Omega^{\alpha}$, we carry the constant $\alpha \leq A$ through the steps and ignore spreading to $\Omega^{1}$,

$$
\begin{aligned}
\alpha^{1 / 2}\left\|\nabla\left(I_{H}\left(\eta v^{\mathrm{f}}\right)\right)\right\|_{L^{2}\left(\Omega^{\alpha} \cap \omega\right)} & \stackrel{19}{\leq} \alpha^{1 / 2} C_{\rho, \kappa}\left\|\nabla\left(\eta v^{\mathrm{f}}\right)\right\|_{L^{2}\left(U_{1}(\omega)\right)} \\
& \leq C_{\rho, \kappa} H^{-1}\left\|A^{1 / 2} v^{\mathrm{f}}\right\|_{L^{2}\left(U_{1}(\omega)\right)}+C_{\rho, \kappa}\left\|A^{1 / 2} \nabla v^{\mathrm{f}}\right\|_{L^{2}\left(U_{1}(\omega)\right)} \\
& \stackrel{222}{\leq} C_{\rho, p, \kappa, \ell}\left\|A^{1 / 2} \nabla v^{\mathrm{f}}\right\|_{L^{2}\left(U_{\ell+1}(\omega)\right)} .
\end{aligned}
$$

The assertion follows from adding the two contributions.

\subsection{Contrast independent localization error bounds}

The proof technique used here follows closely e.g. 14. The first lemma shows that non-local correctors exhibit exponential decay and the second shows that the localization error decays exponentially with increasing patch size $k$ independent of $\alpha$. We use the following elementwise decomposition of $Q v=$ $\sum_{T \in \mathcal{T}_{H}} Q_{T} v$ and $R f=\sum_{T \in \mathcal{T}_{H}} R_{T} f$, where, for all $v^{\mathrm{f}} \in V^{\mathrm{f}}$,

$$
a\left(Q_{T} v, v^{\mathrm{f}}\right)=\int_{T} A \nabla v \cdot \nabla v^{\mathrm{f}} \quad \text { and } \quad a\left(R_{T} v, v^{\mathrm{f}}\right)=\int_{T} f v^{\mathrm{f}} .
$$

With this decomposition, the lemmas below can be applied with $p^{T}=Q_{T} v$ or $p^{T}=R_{T} v$ for any $T \in \mathcal{T}_{H}$ and $v \in V$. 
Lemma 7 (Contrast independent exponential decay of non-local corrector). Let $F_{T}(v) \in V^{\prime}$ with $F_{T}(v)=$ 0 for $v \in V(\Omega \backslash T)$. Let $p^{T} \in V^{\mathrm{f}}$ satisfy, for all $v \in V^{\mathrm{f}}$,

$$
\int_{\Omega} A \nabla p^{T} \cdot \nabla v=F_{T}(v) .
$$

Under Assumptions B, it holds for $k \geq \ell+5$,

$$
\left\|A^{1 / 2} \nabla p^{T}\right\|_{L^{2}\left(\Omega \backslash U_{k}(T)\right)} \leq C_{\rho, p, \kappa, \ell} \theta^{k}\left\|A^{1 / 2} \nabla p^{T}\right\|_{L^{2}(\Omega)},
$$

where $0<\theta<1$ and $C_{\rho, p, \kappa, \ell}$ do not depend on $\alpha, k$ or $H$.

Proof. Let $m=k-\ell-3 \geq 2$. For brevity, we drop a few indices and denote by $U_{m}=U_{m}(T), p=p^{T}$ and $\eta=\eta_{m}^{T}$.

$$
\begin{aligned}
\left\|A^{1 / 2} \nabla p\right\|_{L^{2}\left(\Omega \backslash U_{m}\right)}^{2} & =\int_{\Omega \backslash U_{m}} A \nabla p \cdot \nabla p \leq \int_{\Omega \backslash U_{m-1}} A \nabla p \cdot \eta \nabla p \\
& =\underbrace{\int_{\Omega \backslash U_{m-2}} A \nabla p \cdot \nabla(\eta p)}_{=: \mathrm{I}}-\underbrace{\int_{\Omega \backslash U_{m-1}} A \nabla p \cdot p \nabla \eta}_{=: \mathrm{II}} .
\end{aligned}
$$

For term I, we use that $\int_{\Omega \backslash U_{m-2}} A \nabla p \cdot \nabla\left(\eta p-I_{H}(\eta p)\right)=\int_{\Omega} A \nabla p \cdot \nabla\left(\eta p-I_{H}(\eta p)\right)=F_{T}\left(\eta p-I_{H}(\eta p)\right)=0$, since $\eta p-I_{H}(\eta p)$ has no support in $U_{m-2}(T) \supset T$ (requires $m \geq 2$ ). Since $\operatorname{supp}\left(I_{H}(\eta p)\right) \subset U_{m+1} \backslash U_{m-2}$ we get

$$
\begin{aligned}
& |\mathrm{I}|=\left|\int_{U_{m+1} \backslash U_{m-2}} A \nabla p \cdot \nabla\left(I_{H}(\eta p)\right)\right| \\
& \stackrel{24 \mid}{\leq} C_{\rho, p, \kappa, \ell}\left\|A^{1 / 2} \nabla p\right\|_{L^{2}\left(U_{m+1} \backslash U_{m-2}\right)}\left\|A^{1 / 2} \nabla p\right\|_{L^{2}\left(U_{m+\ell+3} \backslash U_{m-\ell-4}\right)} .
\end{aligned}
$$

For term II, we use that $\operatorname{supp}(\nabla \eta) \subset U_{m} \backslash U_{m-1}$ and $\|\nabla \eta\|_{L^{\infty}(\Omega)} \leq C_{\rho} H^{-1}$ to get

$$
\begin{aligned}
& |\mathrm{II}| \leq C_{\rho} H^{-1}\left\|A^{1 / 2} \nabla p\right\|_{L^{2}\left(U_{m} \backslash U_{m-1}\right)}\left\|A^{1 / 2} p\right\|_{L^{2}\left(U_{m} \backslash U_{m-1}\right)} \\
& \quad \stackrel{22\}}{\leq} C_{\rho, p, \kappa, \ell}\left\|A^{1 / 2} \nabla p\right\|_{L^{2}\left(U_{m} \backslash U_{m-1}\right)}\left\|A^{1 / 2} \nabla p\right\|_{L^{2}\left(U_{m+\ell} \backslash U_{m-\ell-1}\right)} .
\end{aligned}
$$

We obtain, with $c=C_{\rho, p, \kappa, \ell}$,

$$
\begin{aligned}
\left\|A^{1 / 2} \nabla p\right\|_{L^{2}\left(\Omega \backslash U_{m+\ell+3}\right)}^{2} & \left.\leq\left\|A^{1 / 2} \nabla p\right\|_{L^{2}\left(\Omega \backslash U_{m}\right)}^{2} \leq c\left\|A^{1 / 2} \nabla p\right\|_{L^{2}\left(U_{m+\ell+3} \backslash U_{m-\ell-4}\right)}^{2}\right) \\
& =c\left(\left\|A^{1 / 2} \nabla p\right\|_{L^{2}\left(\Omega \backslash U_{m-\ell-4}\right)}^{2}-\left\|A^{1 / 2} \nabla p\right\|_{L^{2}\left(\Omega \backslash U_{m+\ell+3}\right)}^{2}\right) .
\end{aligned}
$$

from which we get the following decay result over $2 \ell+7$ patch layers,

$$
\left\|A^{1 / 2} \nabla p\right\|_{L^{2}\left(\Omega \backslash U_{m+\ell+3}\right)}^{2} \leq \frac{c}{1+c}\left\|A^{1 / 2} \nabla p\right\|_{L^{2}\left(\Omega \backslash U_{m-\ell-4}\right)}^{2} .
$$

Successive application of 26 yields

$$
\left\|A^{1 / 2} \nabla p\right\|_{L^{2}\left(\Omega \backslash U_{m+\ell+3}\right)}^{2} \leq\left(\frac{c}{1+c}\right)^{\lfloor m /(2 \ell+7)\rfloor}\left\|A^{1 / 2} \nabla p\right\|_{L^{2}(\Omega)}^{2} .
$$

The final result is obtained by choosing $\theta=\left(\frac{c}{1+c}\right)^{1 /(4 \ell+14)}$, which is independent of $\alpha$.

Lemma 8 (Contrast independent localization error). Let $F_{T}(v) \in V^{\prime}$ with $F_{T}(v)=0$ for $v \in V(\Omega \backslash T)$. Let $p^{T} \in V^{\mathrm{f}}$ satisfy, for all $v \in V^{\mathrm{f}}$,

$$
\int_{\Omega} A \nabla p^{T} \cdot \nabla v=F_{T}(v)
$$

and $p=\sum_{T \in \mathcal{T}_{H}} p^{T}$. Further, let $p_{k}^{T} \in V^{\mathrm{f}}\left(U_{k}(T)\right)$ satisfy, for all $v \in V^{\mathrm{f}}\left(U_{k}(T)\right)$,

$$
\int_{U_{k}(T)} A \nabla p_{k}^{T} \cdot \nabla v=F_{T}(v)
$$


and $p_{k}=\sum_{T \in \mathcal{T}_{H}} p_{k}^{T}$. Then, for $k \geq \ell+5$,

$$
\left\|A^{1 / 2} \nabla\left(p-p_{k}\right)\right\|_{L^{2}(\Omega)} \leq C_{\rho, p, \kappa, \ell} k^{d / 2} \theta^{k}\left(\sum_{T \in \mathcal{T}_{H}}\left\|A^{1 / 2} \nabla p^{T}\right\|_{L^{2}(\Omega)}^{2}\right)^{1 / 2},
$$

where $0<\theta<1$ and $C_{\rho, p, \kappa, \ell}$ do not depend on $\alpha, k$ or $H$.

Proof. Let $z=p-p_{k}=\sum_{T \in \mathcal{T}_{H}}\left(p^{T}-p_{k}^{T}\right)$. Since, for all $T, \tilde{z}=\eta_{k+2}^{T} z-I_{H}\left(\eta_{k+2}^{T} z\right) \in V^{\mathrm{f}}$, with $F_{T}(\tilde{z})=0$ and $p_{k}^{T}$ and $\tilde{z}$ lack common support, we have $\int_{\Omega} A \nabla \tilde{z} \cdot \nabla\left(p^{T}-p_{k}^{T}\right)=0$ and we get

$$
\begin{aligned}
\left\|A^{1 / 2} \nabla z\right\|_{L^{2}(\Omega)}^{2}= & \sum_{T \in \mathcal{T}_{H}} \int_{\Omega} A \nabla\left(\left(1-\eta_{k+2}^{T}\right) z+I_{H}\left(\eta_{k+2}^{T} z\right)\right) \cdot \nabla\left(p^{T}-p_{k}^{T}\right) \\
\leq & \sum_{T \in \mathcal{T}_{H}}(\underbrace{\left\|A^{1 / 2} \nabla\left(\left(1-\eta_{k+2}^{T}\right) z\right)\right\|_{L^{2}(\Omega)}}_{=: \mathrm{I}}+\underbrace{\left\|A^{1 / 2} \nabla I_{H}\left(\eta_{k+2}^{T} z\right)\right\|_{L^{2}(\Omega)}}_{=: \mathrm{II}}) . \\
& \cdot \underbrace{\left\|A^{1 / 2} \nabla\left(p^{T}-p_{k}^{T}\right)\right\|_{L^{2}(\Omega)}}_{=: \mathrm{III}} .
\end{aligned}
$$

For term I, we have

$$
\begin{aligned}
\left\|A^{1 / 2} \nabla\left(\left(1-\eta_{k+2}^{T}\right) z\right)\right\|_{L^{2}(\Omega)} & \leq\left\|A^{1 / 2} \nabla\left(\left(1-\eta_{k+2}^{T}\right) z\right)\right\|_{L^{2}\left(U_{k+2}(T)\right)} \\
& \leq C_{\rho} H^{-1}\left\|A^{1 / 2} z\right\|_{L^{2}\left(U_{k+2}(T) \backslash U_{k+1}(T)\right)}+\left\|A^{1 / 2} \nabla z\right\|_{L^{2}\left(U_{k+2}(T)\right)} \\
& \leq C_{\rho, p, \kappa, \ell}\left\|A^{1 / 2} \nabla z\right\|_{L^{2}\left(U_{k+\ell+2}(T)\right)} .
\end{aligned}
$$

For term II, we have

$$
\begin{aligned}
\left\|A^{1 / 2} \nabla I_{H}\left(\eta_{k+2}^{T} z\right)\right\|_{L^{2}(\Omega)} & =\left\|A^{1 / 2} \nabla I_{H}\left(\eta_{k+2}^{T} z\right)\right\|_{L^{2}\left(U_{k+3}(T) \backslash U_{k+1}(T)\right)} \\
& \stackrel{24]}{\leq} C_{\rho, p, \kappa, \ell}\left\|A^{1 / 2} \nabla z\right\|_{L^{2}\left(U_{k+\ell+5}(T)\right)} .
\end{aligned}
$$

For factor III, we note that $\tilde{p}_{k}^{T}=\left(1-\eta_{k-1}^{T}\right) p^{T}-I_{H}\left(\left(1-\eta_{k-1}^{T}\right) p^{T}\right) \in V^{\mathrm{f}}\left(U_{k}(T)\right)$ and that $p_{k}^{T}$ is bestapproximation of $p^{T}$ in $V^{\mathrm{f}}\left(U_{k}(T)\right)$ in energy norm, why

$$
\begin{aligned}
\left\|A^{1 / 2} \nabla\left(p^{T}-p_{k}^{T}\right)\right\|_{L^{2}(\Omega)} & \leq\left\|A^{1 / 2} \nabla\left(p^{T}-\tilde{p}_{k}^{T}\right)\right\|_{L^{2}(\Omega)} \\
& \leq\left\|A^{1 / 2} \nabla\left(\eta_{k-1}^{T} p^{T}-I_{H}\left(\eta_{k-1}^{T} p^{T}\right)\right)\right\|_{L^{2}(\Omega)} \\
& \leq C_{\rho, p, \kappa, \ell}\left\|A^{1 / 2} \nabla p^{T}\right\|_{L^{2}\left(\Omega \backslash U_{k-\ell-2}(T)\right)}+ \\
& \left.+\| A^{1 / 2} \nabla I_{H}\left(\eta_{k-1}^{T} p^{T}\right)\right) \|_{\left.L^{2}\left(U_{k}(T) \backslash U_{k-3}(T)\right)\right)} \\
& \leq C_{\rho, p, \kappa, \ell}\left\|A^{1 / 2} \nabla p^{T}\right\|_{L^{2}\left(\Omega \backslash U_{k-\ell-3}(T)\right)} \\
& \leq C_{\rho, p, \kappa, \ell} \theta^{k-\ell-3}\left\|A^{1 / 2} \nabla p^{T}\right\|_{L^{2}(\Omega)} .
\end{aligned}
$$

Collecting the terms and, we continue from equation 27) using Hölder's inequality and obtain

$$
\begin{aligned}
\left\|A^{1 / 2} \nabla z\right\|_{L^{2}(\Omega)}^{2} & \leq C_{\rho, p, \kappa, \ell} \theta^{k-\ell-3} \sum_{T \in \mathcal{T}_{H}}\left\|A^{1 / 2} \nabla z\right\|_{L^{2}\left(U_{k+\ell+5}(T)\right)}\left\|A^{1 / 2} \nabla p^{T}\right\|_{L^{2}(\Omega)} \\
& \leq C_{\rho, p, \kappa, \ell}(k+\ell+5)^{d / 2} \theta^{k-\ell-3}\left\|A^{1 / 2} \nabla z\right\|_{L^{2}(\Omega)}\left(\sum_{T \in \mathcal{T}_{H}}\left\|A^{1 / 2} \nabla p^{T}\right\|_{L^{2}(\Omega)}^{2}\right)^{1 / 2} .
\end{aligned}
$$

The lemma follows, since $\ell+5 \leq k$. 


\subsection{Error bounds for localized multiscale method}

As a result of the contrast independent localization error, we get the following error bounds for the localized multiscale method, without and with right hand side correction.

Theorem 9 (Error bound without right hand side correction). If $u_{H, k}^{\mathrm{ms}}$ is computed as described in Section 2.2, then

$$
\left\|u-u_{H, k}^{\mathrm{ms}}\right\| \leq \leq C_{\Omega, \rho, p, \kappa, \ell}\left(\alpha^{-1} k^{d / 2} \theta^{k}\|f\|_{L^{2}(\Omega)}+H\left\|A^{-1 / 2} f\right\|_{L^{2}(\Omega)}\right),
$$

where $C_{\Omega, \rho, p, \kappa, \ell}$ and $0<\theta<1$ are independent of $\alpha, k$, and $H$.

Proof. Galerkin orthogonality implies $\left\|\left|u-u_{H, k}^{\mathrm{ms}}\|\| \leq\left\|\mid u-v_{H, k}^{\mathrm{ms}}\right\| \|\right.\right.$ for any $v_{H, k}^{\mathrm{ms}} \in V_{H, k}^{\mathrm{ms}}$. We decompose $u=u_{H}^{\mathrm{ms}}+u^{\mathrm{f}}=u_{H}-Q u_{H}+u^{\mathrm{f}}$ and choose $v_{H, k}^{\mathrm{ms}}=u_{H}-Q_{k} u_{H}$. Then by Lemma 8

$$
\begin{aligned}
\left\|u-u_{H, k}^{\mathrm{ms}}\right\| & \leq\|\| u_{H}^{\mathrm{ms}}-v_{H, k}^{\mathrm{ms}}+u^{\mathrm{f}} \mid\|\leq\| Q u_{H}-Q_{k} u_{H}\|+\|\left\|u^{\mathrm{f}}\right\| \\
& \leq C_{\rho, p, \kappa, \ell} k^{d / 2} \theta^{k}\left(\sum_{T \in \mathcal{T}_{H}}\left\|Q_{T} u_{H}\right\|^{2}\right)^{1 / 2}+\|\| u^{\mathrm{f}} \| .
\end{aligned}
$$

For the first term, using 19, , we get

$$
\begin{aligned}
\left(\sum_{T \in \mathcal{T}_{H}}\left\|Q_{T} u_{H}\right\|^{2}\right)^{1 / 2} & \leq\left(\sum_{T \in \mathcal{T}_{H}}\left\|A^{1 / 2} \nabla u_{H}\right\|_{L^{2}(T)}^{2}\right)^{1 / 2}=\left\|u_{H}\right\|=\left\|I_{H}\left(u_{H}^{\mathrm{ms}}\right)\right\| \leq\left\|\nabla I_{H}\left(u_{H}^{\mathrm{ms}}\right)\right\|_{L^{2}(\Omega)} \\
& \leq C_{\rho, \kappa}\left\|\nabla u_{H}^{\mathrm{ms}}\right\|_{L^{2}(\Omega)} \leq \alpha^{-1 / 2} C_{\rho, \kappa}\left\|u_{H}^{\mathrm{ms}}\right\| \leq \alpha^{-1} C_{\Omega, \rho, \kappa}\|f\|_{L^{2}(\Omega)} .
\end{aligned}
$$

For the second term, we get

$$
\begin{aligned}
\left\|u^{\mathrm{f}}\right\|^{2} & =\left(A^{-1 / 2} f, A^{1 / 2} u^{\mathrm{f}}\right) \leq\left\|A^{-1 / 2} f\right\|_{L^{2}(\Omega)}\left\|A^{1 / 2} u^{\mathrm{f}}\right\|_{L^{2}(\Omega)} \\
& \leq\left\|A^{-1 / 2} f\right\|_{L^{2}(\Omega)}\left(\alpha^{1 / 2}\left\|u^{\mathrm{f}}-I_{H} u^{\mathrm{f}}\right\|_{L^{2}\left(\Omega^{\alpha}\right)}+\left\|u^{\mathrm{f}}\right\|_{L^{2}\left(\Omega^{1}\right)}\right) \\
& \quad C_{\rho, p, \kappa, \ell} H\left\|A^{-1 / 2} f\right\|_{L^{2}(\Omega)}\left\|A^{1 / 2} \nabla u^{\mathrm{f}}\right\|_{L^{2}(\Omega)} \\
& =C_{\rho, p, \kappa, \ell} H\left\|A^{-1 / 2} f\right\|_{L^{2}(\Omega)}\left\|u^{\mathrm{f}}\right\|,
\end{aligned}
$$

which proves the lemma.

Theorem 10 (Error bound with right hand side correction). If $u_{H, k}=u_{H, k}^{\mathrm{ms}, \mathrm{rhs}}+u_{k}^{\mathrm{f}}$ is computed as described in Section 2.3, then

$$
\left\|u-u_{H, k}\right\| \leq C_{\Omega, \rho, p, \kappa, \ell} k^{d / 2} \theta^{k}\left(\alpha^{-1}\|f\|_{L^{2}(\Omega)}+H\left\|A^{-1 / 2} f\right\|_{L^{2}(\Omega)}\right),
$$

where $C_{\Omega, \rho, p, \kappa, \ell}$ and $0<\theta<1$ are independent of $\alpha, k$, and $H$. The second term is dominated by the first, but kept for easy comparison with the previous lemma.

Proof. Galerkin orthogonality implies $\left\|\mid u_{H}^{\mathrm{ms}}-u_{H, k}^{\mathrm{ms}, \mathrm{rhs}}\right\| \leq\|\| u_{H}^{\mathrm{ms}}-v_{H, k}^{\mathrm{ms}}\|\|$ for any $v_{H, k}^{\mathrm{ms}} \in V_{H, k}^{\mathrm{ms}}$. We decompose $u=u_{H}^{\mathrm{ms}}+u^{\mathrm{f}}=u_{H}-Q u_{H}+u^{\mathrm{f}}$ and choose $v_{H, k}^{\mathrm{ms}}=u_{H}-Q_{k} u_{H}$. Then by Lemma 8

$$
\begin{aligned}
\left\|u-u_{H, k}\right\| \mid & \leq\|\| u_{H}^{\mathrm{ms}}-u_{H, k}^{\mathrm{ms}, \mathrm{rhs}} \mid\|+\| u^{\mathrm{f}}-u_{k}^{\mathrm{f}}\|\| \leq\left\|Q u_{H}-Q_{k} u_{H}\right\|+\left\|R f-R_{k} f\right\| \| \\
& \leq C_{\rho, p, \kappa, \ell} k^{d / 2} \theta^{k}\left(\left(\sum_{T \in \mathcal{T}_{H}}\left\|Q_{T} u_{H}\right\|^{2}\right)^{1 / 2}+\left(\sum_{T \in \mathcal{T}_{H}}\left\|R_{T} f\right\|^{2}\right)^{1 / 2}\right) .
\end{aligned}
$$

The first term is bounded as in Theorem 9. For the second term, we use 22 in Lemma 5 and get

$$
\left\|R_{T} f\right\|^{2} \leq\left\|A^{-1 / 2} f\right\|_{L^{2}(T)}\left\|A^{1 / 2} R_{T} f\right\|_{L^{2}(T)} \leq C_{\rho, p, \kappa, \ell} H\left\|A^{-1 / 2} f\right\|_{L^{2}(T)}\left\|R_{T} f\right\| \|,
$$

which concludes the proof. 


\section{$5 \quad$ Numerical experiments}

We present three numerical experiments with different coefficients to illustrate how the full error of the solution with right hand side correction (Theorem 10) depends on the choice of patch size $k$ and contrast $\alpha$ for six different interpolation operators. The results are discussed in Section 5.4

\subsection{Mesh and coefficient}

The computational domain is $\Omega=[0,1]^{2}$ in all numerical experiments and we use the mesh family shown in Figure 10. In addition to the coarse mesh $\mathcal{T}_{H}$ parametrized by $H$, we use a fine mesh $\mathcal{T}_{h}$ parametrized by a fine mesh parameter $h<H$. Although the method above is described in terms an infinite dimensional full space $V$, we use the fine mesh to discretize $V$ into a $\mathcal{P} 1 \mathrm{FE}$ space $V_{h}$, with basis functions $\phi_{h, x}$ for nodes $x$ and $h=2^{-10}$. The coefficient $A$ is defined as a piecewise constant function on the fine mesh, taking either value 1 or $\alpha$ on every fine element. In all figures depicting a coefficient, black corresponds to $\Omega^{1}$ and white to $\Omega^{\alpha}$.

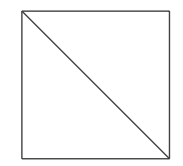

(a) Coarsest mesh, $H=1$.

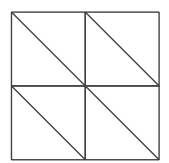

(b) One refinement, $H=1 / 2$.

Figure 10: Family $\mathcal{T}_{H}$ of triangulations of the unit square.

\subsection{Interpolation operators}

The choice of interpolation operator $\mathcal{I}_{H}$ determines the properties of the multiscale method. Besides $I_{H}$ and $I_{H, 1}$, we study four additional interpolation operators. We comment on all of them below.

\subsubsection{Operators $I_{H}$ and $I_{H, 1}$}

The formal definitions of $I_{H}$ and $I_{H, 1}$ can be found in Section 3.3 and are based on the subdomains $\omega_{i}^{1}$ through the integration domains $\sigma_{i}$. The procedure is reversed in our implementation: $\omega_{i}^{1}$ is implicitly defined from $\sigma_{i}$, which is determined in the following procedure. For each node $z_{i}$ :

1. If $A=\alpha$ in all fine elements neighboring $z_{i}$ it is a class II node and $\sigma_{i}=\Sigma_{\delta}\left(z_{i}\right)$, i.e. $1 / 4$ scaling of a node-patch for $I_{H}$, and full node-patch for $I_{H, 1}$.

2. Otherwise the node is a class I node. Select any element neighboring $z_{i}$ with $A=1$. Let $\sigma_{i}$ be all fine elements within the node patch for which there is an edge-incident path of elements with $A=1$ to the selected element. This guarantees that $\sigma_{i}$ is always a connected subset of $\Omega^{1}$.

As a result of this procedure, whether Assumption B is satisfied or not depends on the node placement.

\subsubsection{Node patch Scott-Zhang $I_{H}^{\mathrm{SZ}}$}

The node patch Scott-Zhang quasi-interpolation is denoted by $I_{H}^{\mathrm{SZ}}: V \rightarrow V_{H}$ and is defined, for every free node $z_{i} \in \mathcal{N}_{H}^{\text {free }}$, by

$$
\left(I_{H}^{\mathrm{SZ}} v\right)\left(z_{i}\right)=N_{i}(v)
$$

with $\sigma_{i}=U\left(z_{i}\right)$. Here, $N_{i}$ is defined as in Section 5.2.2. This operator was introduced in 25], where also the stability and approximability properties asserted in Assumption A were shown. This operator is a Clément-type quasi-interpolation operators similar to those used in many previous works on the LOD technique, e.g. [1, 4, 10, 12, 13, 19, 22, 


\subsubsection{Nodal interpolation $I_{H}^{\text {nodal }}$}

We denote the nodal interpolation operator by $I_{H}^{\text {nodal }}: C^{0}(\Omega) \rightarrow V_{H}$ and it is defined by

$$
\left(I_{H}^{\text {nodal }} v\right)(z)=v(z)
$$

for all free nodes $z \in \mathcal{N}_{H}^{\text {free }}$. Note that this operator is not defined for all functions in $V$ for $d \geq 2$, and hence does not fulfill Assumption A. Nevertheless, it is of interest to include, since it is easy to implement and it is well-defined if $V$ is a finite element space on a very fine mesh. This is the case in all numerical experiments performed in this paper.

\subsubsection{A-weighted projection $I_{H}^{A}$}

The A-weighted projective quasi-interpolation operator is denoted by $I_{H}^{A}: V \rightarrow V_{H}$ and is defined by, for all free nodes $z \in \mathcal{N}_{H}^{\text {free }}$,

$$
\left(I_{H}^{A} v\right)(z)=\left(P_{z} v\right)(z),
$$

where $P_{z}$ is the projection $\left.P_{z} v \in S_{H}\right|_{U(z)}$ (functions in $S_{H}$ restricted to node patch $U(z)$ ) such that $\int_{U(z)} A P_{z} v w=\int_{U(z)} A v w$ for all $\left.w \in S_{H}\right|_{U(z)}$. This operator was used in 23] to define the fine space and to show contrast independent localization error bounds under quasi-monotonicity assumptions on the coefficient distribution within the node patches. This assumption can be restrictive for coarse meshes.

\subsubsection{A-weighted projection with quasi-monotonicity $I_{H}^{A, \mathrm{qm}}$}

The A-weighted projective quasi-interpolation operator with guaranteed quasi-monotonicity is denoted by $I_{H}^{A, \mathrm{qm}}: V \rightarrow V_{H}$ and is defined like $I_{H}^{A}$, but with the node patch $U(z)$ replaced by a connected subset of $U(z)$ that contains $z$ and has (type- $(d-1))$ quasi-monotone coefficient distribution. See e.g. 23] for the definition of quasi-monotone coefficient distribution.

\subsection{Experiment: Full error versus patch size and contrast}

Below, we present three different coefficient geometries $\Omega^{1}$ and study the full error between a reference solution on the finest grid and the solution from the localized multiscale method with right hand side correction while varying interpolation operator, patch size and contrast. In all experiments in this subsection, we let $H=2^{-4}$ and solve the right hand side corrected solution $u_{H, k}$ for $\mathcal{I}_{H} \in$ $\left\{I_{H}^{\mathrm{SZ}}, I_{H}^{\text {nodal }}, I_{H}, I_{H, 1}, I_{H}^{A}, I_{H}^{A, \mathrm{qm}}\right\}, k=1, \ldots, 6$, and $\alpha=10^{-1}, 10^{-2}, \ldots, 10^{-6}$. We compute a reference solution $u_{h}$ (with $h=2^{-10}$ ) and the relative error $\left\|u_{h}-u_{H, k}\right\| /\left\|u_{h}\right\|$ for each tuple $\left(\mathcal{I}_{H}, \Omega^{1}, k, \alpha\right)$. The corresponding error bound for $I_{H}$ can be found in Theorem 10

\subsubsection{Stripes}

Here $A$ is defined as illustrated in Figure 11a. The stripes are located at distance 1/16 from each other and their width is $1 / 128$. With $H=2^{-4}$ there is a node in each stripe and Assumption B is satisfied. We let $f=\chi_{[1 / 4,3 / 4]^{2}}(\chi$ is the indicator function) and use homogeneous Dirichlet boundary conditions on the full boundary. The relative errors for the the different interpolation operators and contrasts are presented in Figure 11. Quasi-monotonicity is not satisfied for this mesh, but would require two additional mesh refinements to be fulfilled.

\subsubsection{Random balls}

Here $A$ was constructed in the following manner. Starting with a white background, for every node in the coarse mesh $\left(H=2^{-4}\right)$, randomly (chance 50\%) place a black ball of random radius between $1 / 128$ and 8/128 with center at the node. $A$ is defined according to the convention of black representing $\Omega^{1}$ and white representing $\Omega^{\alpha}$. See Figure 12a We let $f=\chi_{[1 / 4,3 / 4]^{2}}$ and use homogeneous Dirichlet boundary conditions on the full boundary. The relative errors for the the different interpolation operators and contrasts are presented in Figure 12 . We note that Assumption B is satisfied since there is a node in each ball. Quasi-monotonicity is not satisfied. 


\subsubsection{Random field}

Here $A$ was constructed by a random process with spatial correlation. See Figure 13a We use $f=\phi_{h, x}$ for $x=(1 / 2,1 / 8)$ and impose homogeneous Neumann boundary conditions on all edge segments except the right-most $\left(x_{2}=1\right)$, where we impose homogeneous Dirichlet boundary conditions. The relative errors for the the different interpolation operators and contrasts are presented in Figure 13 In this case, neither Assumption B nor quasi-monotonicity holds for $H=2^{-4}$.

\subsection{Discussion}

We discuss the numerical results in the light of the theoretical findings from previous sections. Although the localization error in Lemma 8 is independent of the contrast, we note that the contrast factor $\alpha^{-1}$ nevertheless enters the full error bound in Theorem 10

$$
\left\|u-u_{H, k}\right\| \leq C_{\Omega, \rho, p, \kappa, \ell} \alpha^{-1} k^{d / 2} \theta^{k}\|f\|_{L^{2}(\Omega)} .
$$

The contrast factor $\alpha^{-1 / 2}$ also enters the following bound of the energy norm of the true solution

$$
\|u\|^{2} \leq\|u\|_{L^{2}(\Omega)} \cdot\|f\|_{L^{2}(\Omega)} \leq C_{\Omega} \alpha^{-1 / 2}\|f\|_{L^{2}(\Omega)} \cdot\|u\| \| .
$$

Thus, assuming the solution norm is proportional to this bound, the relative error plotted in the figures behaves like

$$
\left\|u-u_{H, k}\right\| /\|u\| \approx C_{\Omega, \rho, p, \kappa, \ell} \alpha^{-1 / 2} k^{d / 2} \theta^{k} .
$$

We emphasize that $C_{\Omega, \rho, p, \kappa, \ell}$ and $0<\theta<1$ are proved to be independent of $\alpha$ for $I_{H}$ and $I_{H}^{A}$ (under different assumptions), and we can expect to observe the contrast influencing the plotted relative errors by a factor at most $\alpha^{-1 / 2}$. In particular, the decay rate of the relative error in terms of patch size $k$ is independent of $\alpha$.

The following discussion is based on the results from the experiments, shown in Figures 11, 13 . We start by a few interpolation operator specific observations based on all three experiments.

- The contrast problem is clearly visible for the full node patch Scott-Zhang interpolation operator $I_{H}^{\mathrm{SZ}}$. It has a clear contrast dependent error for all three coefficients. The decay rate deteriorates as $\alpha \rightarrow 0$ in all cases.

- We note that the novel interpolation operator $I_{H}$ shows contrast independent decay rates in all cases. It is interesting to note though, that $I_{H, 1}$ (where $\delta=1$ instead of $1 / 4$ ) shows better decay rates for all coefficients. This suggests that there is room for improving the theoretical results to include this kind of operator (see Remark 1).

- The error from using the nodal interpolation operator $I_{H}^{\text {nodal }}$ appears not to be contrast dependent, but the decay rate is low (except for the stripe coefficient, discussed below). The nodal interpolation operator is similar to $I_{H, \delta}$ with $\delta=h / H=2^{-6}$, which satisfies the presented theory on contrast independence, albeit with very low $L^{2}$-stability (large $\kappa$ ). This is probably what deteriorates the decay rate, but still keeps it contrast independent.

Next, we discuss the results on coefficient specific basis.

- For the stripes coefficient in Figure 11 all operators except $I_{H}^{\mathrm{SZ}}$ show good decay properties. The nodal interpolation operator $I_{H}^{\text {nodal }}$ shows an inverse relationship between error and contrast than what is generally expected. One interpretation is that when the contrast is high, this coefficient effectively constitute a number of weakly coupled 1D-problems. The nodal interpolation operator then leads to good localization according to the total element localization in 1D, discussed in e.g. 17. This also suggests that subdimensional channels (e.g. cracks, faults or fibers) can be handled accurately by this kind of node variables. The smoothing interpolation operator $I_{H}^{\mathrm{SZ}}$ seems to be particularly ill-suited for this kind of coefficient.

- The random balls coefficient in Figure 12 satisfies Assumption B, however the quasi-monotonicity assumption does not hold. We can see that the decay rate of $I_{H}^{A}$ indeed depends on the contrast, while $I_{H}^{\text {nodal }}, I_{H}, I_{H, 1}$ and $I_{H}^{A, \mathrm{qm}}$ enjoy contrast independent decay rates. It is interesting to note 
that the modification of $I_{H}^{A}$ to $I_{H}^{A, \mathrm{qm}}$ (by selecting the integration domain to guarantee quasimonotonicity) was sufficient to remove the contrast dependent decay. The operator $I_{H}$ shows contrast independent decay rate with a better rate than $I_{H}^{\text {nodal }}$, but it appears to suffer from similar deterioration of decay rate as $I_{H}^{\text {nodal }}$.

- The random field coefficient in Figure 13 satisfies neither Assumption B nor quasi-monotonicity. Despite this, the numerical results are similar to those for the random balls. Although the assumptions are not satisfied, the heavily contrast dependent error for $I_{H}^{\mathrm{SZ}}$ in this experiment suggests that there can still be a gain in accuracy using the ideas presented here for coefficients not strictly covered by the assumptions. 


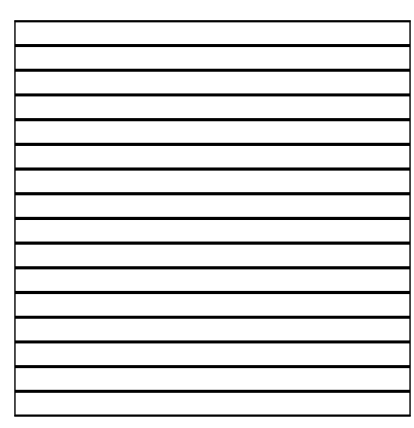

(a) Coefficient

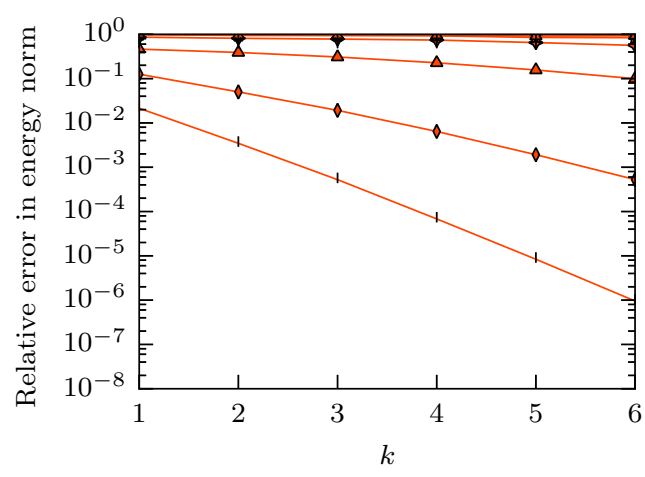

(b) $I_{H}^{\mathrm{SZ}}$

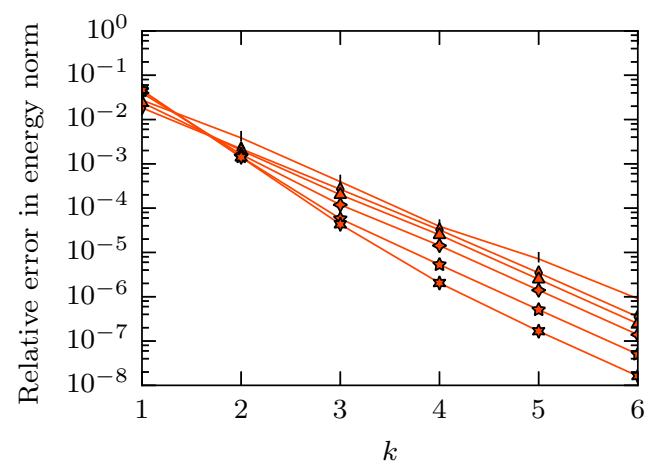

(d) $I_{H}$

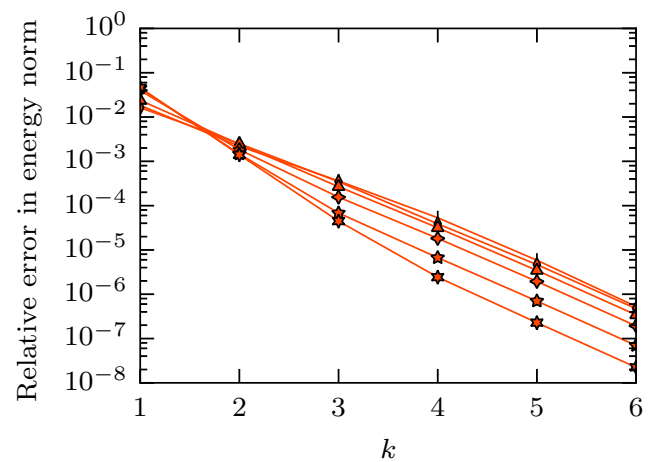

(f) $I_{H}^{A}$

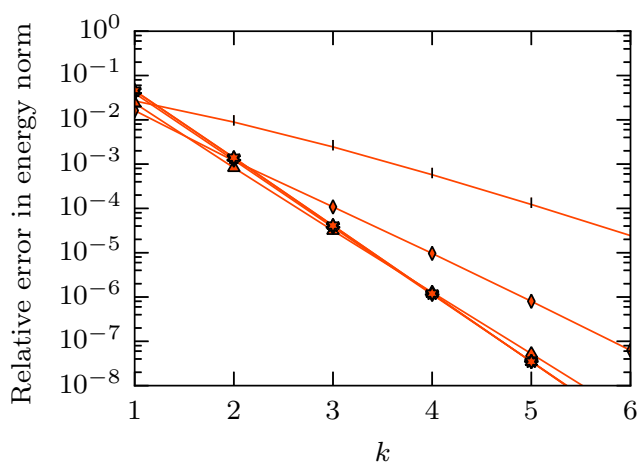

(c) $I_{H}^{\text {nodal }}$

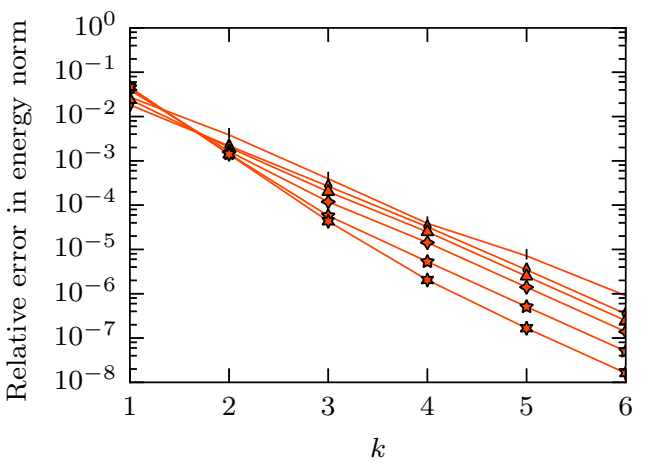

(e) $I_{H, 1}$

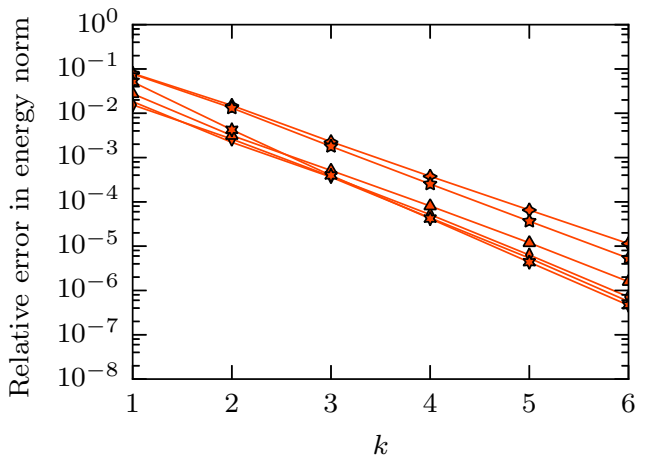

(g) $I_{H}^{A, \mathrm{qm}}$

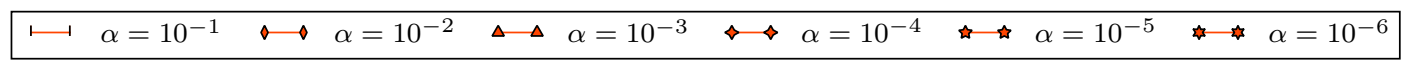

Figure 11: Error versus $k, H=2^{-4}, h=2^{-10}$, stripes coefficient. 


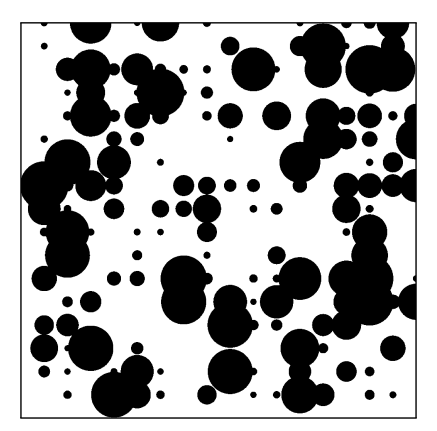

(a) Coefficient

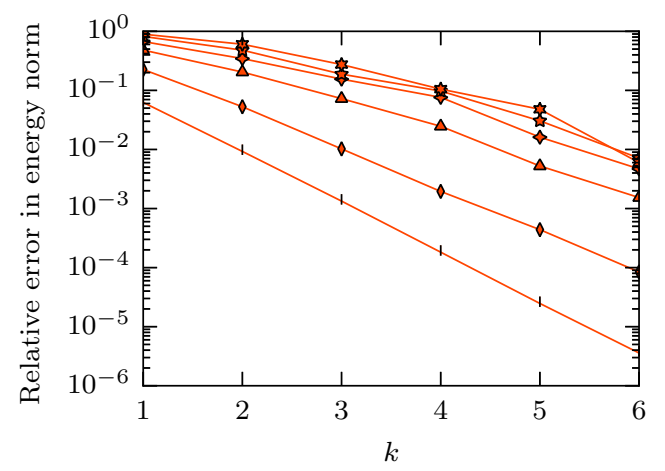

(b) $I_{H}^{\mathrm{SZ}}$

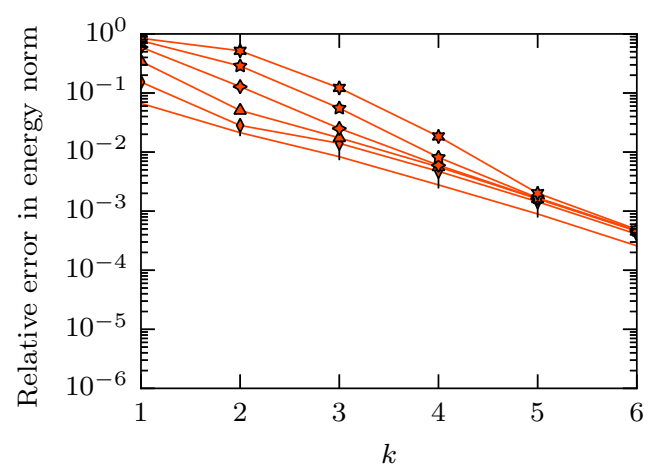

(d) $I_{H}$

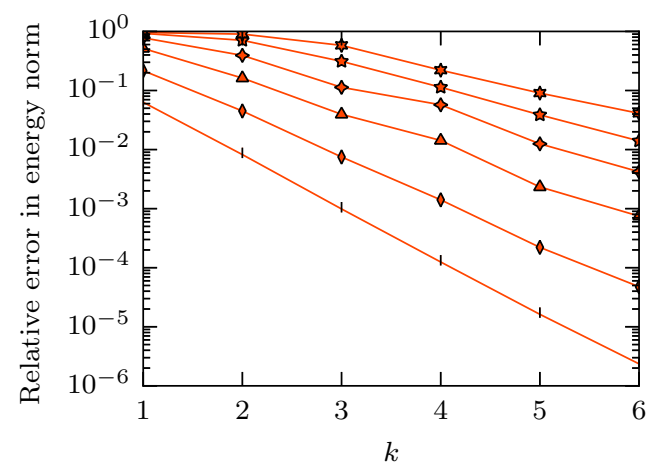

(f) $I_{H}^{A}$

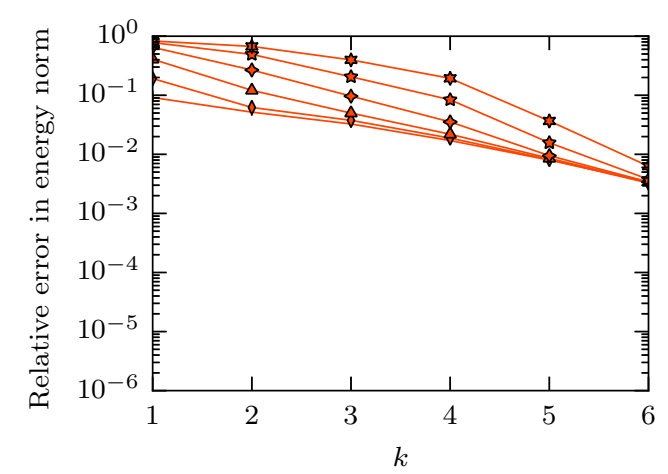

(c) $I_{H}^{\text {nodal }}$

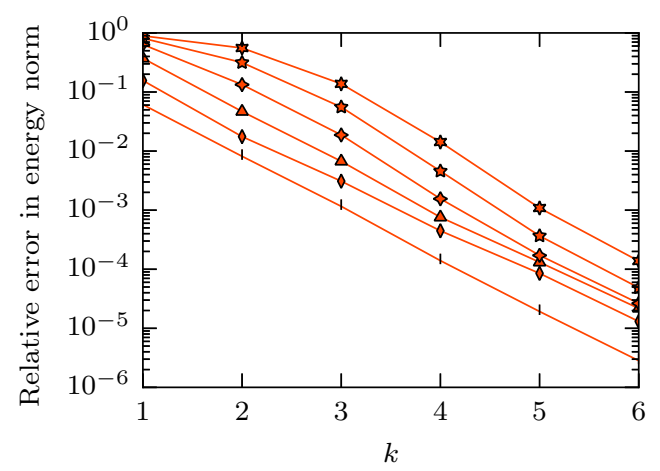

(e) $I_{H, 1}$

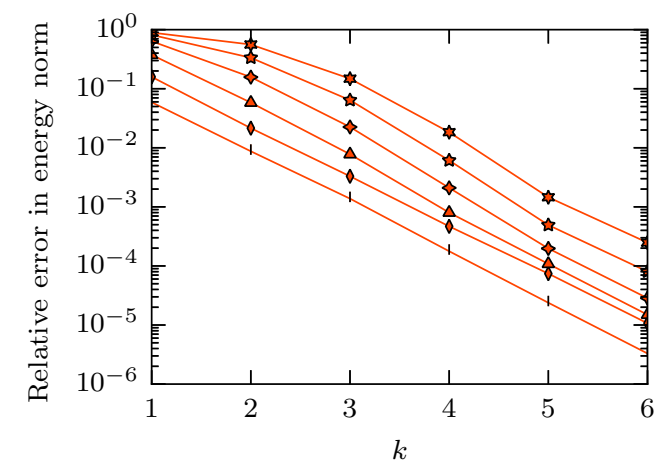

(g) $I_{H}^{A, \mathrm{qm}}$

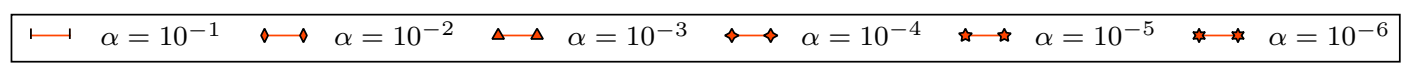

Figure 12: Error versus $k, H=2^{-4}, h=2^{-10}$, random balls coefficient. 


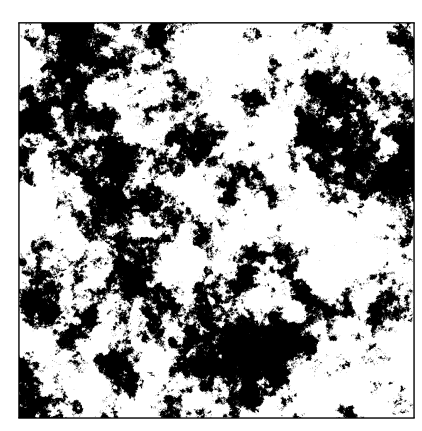

(a) Coefficient

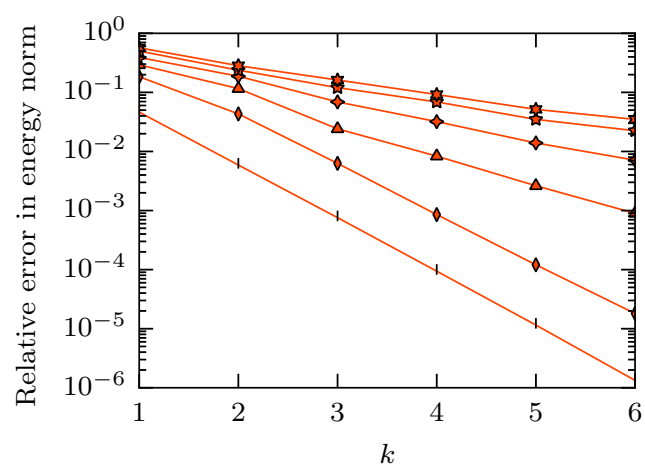

(b) $I_{H}^{\mathrm{SZ}}$

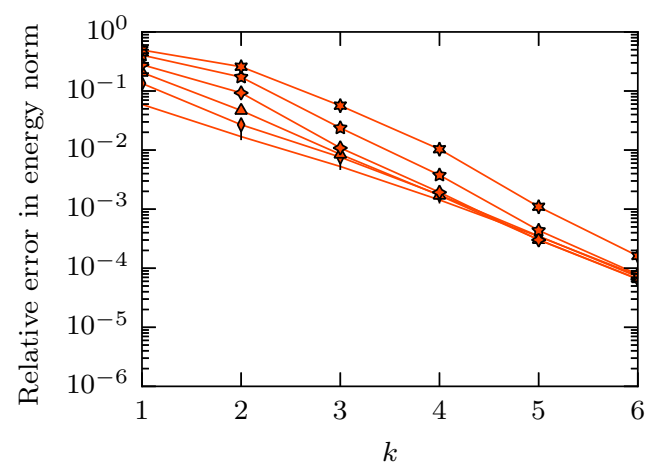

(d) $I_{H}$

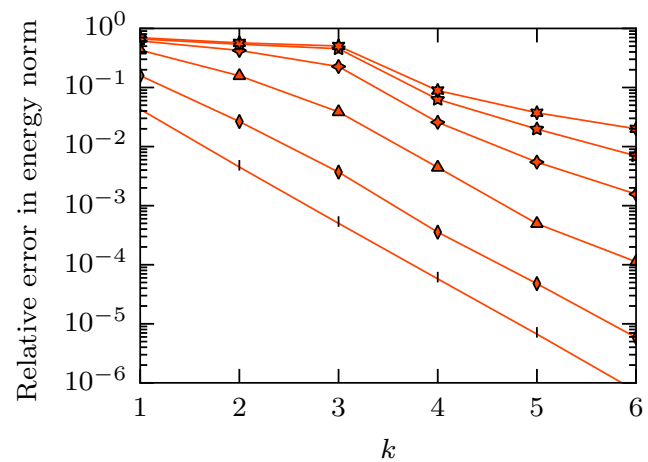

(f) $I_{H}^{A}$

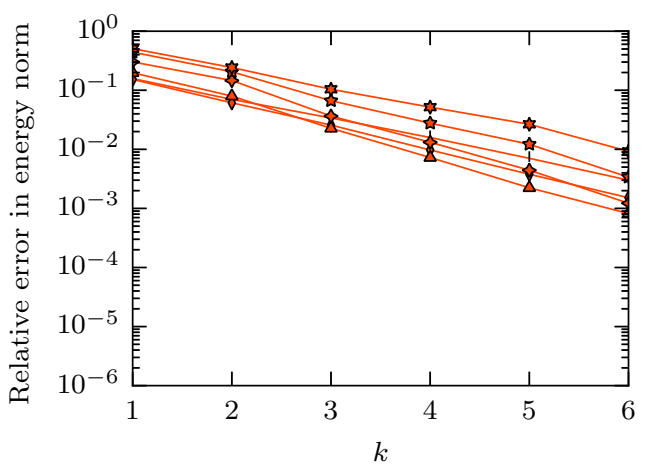

(c) $I_{H}^{\text {nodal }}$

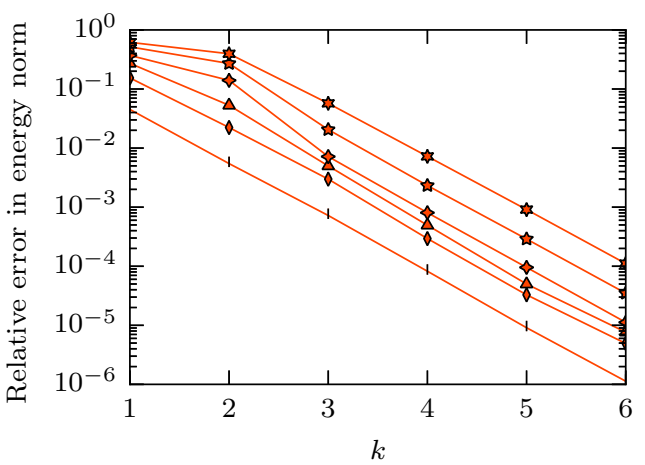

(e) $I_{H, 1}$

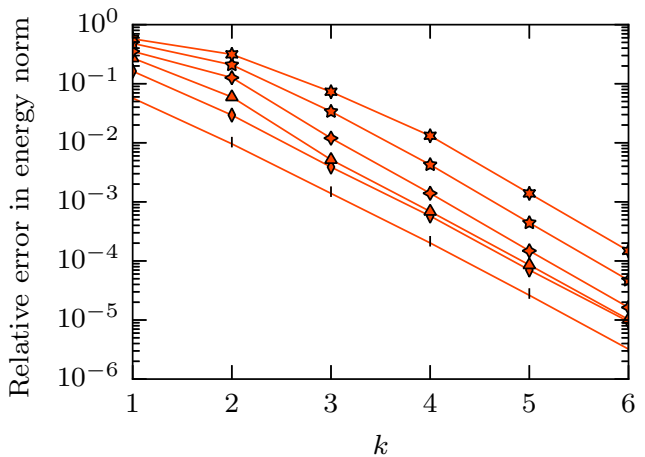

(g) $I_{H}^{A, \mathrm{qm}}$

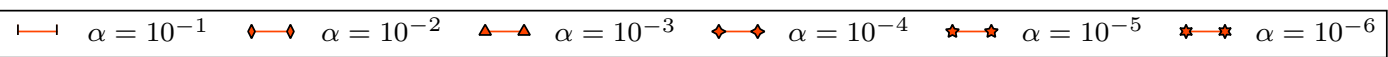

Figure 13: Error versus $k, H=2^{-4}, h=2^{-10}$, random field coefficient. 


\section{References}

[1] A. Abdulle and P. Henning. Localized orthogonal decomposition method for the wave equation with a continuum of scales. Math. Comp., 2016.

[2] M. Bebendorf. Low-rank approximation of elliptic boundary value problems with high-contrast coefficients. SIAM J. Numer. Anal., 48(2):932-949, 2016.

[3] L. Berlyand and H. Owhadi. Flux norm approach to finite dimensional homogenization approximations with non-separated scales and high contrast. Arch. Ration. Mech. Anal., 198(2):677-721, 2010 .

[4] D. L. Brown and D. Peterseim. A multiscale method for porous microstructures. Multiscale Model. Simul., 14(3):1123-1152, 2016.

[5] C.-C. Chu, I. G. Graham, and T.-Y. Hou. A new multiscale finite element method for high-contrast elliptic interface problems. Math. Comp., 79:1915-1955, 2010.

[6] T. Dupont and R. Scott. Polynomial approximation of functions in Sobolev spaces. Math. Comp., 34:441-463, 1980.

[7] W. E and B. Engquist. The heterogeneous multiscale methods. Commun. Math. Sci., 1(1):87-132, 2003.

[8] Y. Efendiev, J. Galvis, and T. Y. Hou. Generalized multiscale finite element methods (GMsFEM). J. Comput. Phys., 251:116 - 135, 2013.

[9] Y. Efendiev, J. Galvis, and X.-H. Wu. Multiscale finite element methods for high-contrast problems using local spectral basis functions. J. Comput. Phys., 230(4):937 - 955, 2011.

[10] D. Elfverson, V. Ginting, and P. Henning. On multiscale methods in Petrov-Galerkin formulation. Numer. Math., 131(4):643-682, 2015.

[11] C. Engwer, P. Henning, A. Målqvist, and D. Peterseim. Efficient implementation of the localized orthogonal decomposition method. arXiv:1602.01658, 2016.

[12] D. Gallistl and D. Peterseim. Stable multiscale Petrov-Galerkin finite element method for high frequency acoustic scattering. Comput. Methods Appl. Mech. Eng., 295:1-17, 2015.

[13] P. Henning and A. Målqvist. Localized orthogonal decomposition techniques for boundary value problems. SIAM J. Sci. Comput., 36(4):A1609-A1634, 2014.

[14] P. Henning, P. Morgenstern, and D. Peterseim. Multiscale partition of unity. In M. Griebel and M. A. Schweitzer, editors, Meshfree Methods for Partial Differential Equations VII, volume 100 of Lecture Notes in Computational Science and Engineering, pages 185-204. Springer International Publishing, 2015.

[15] T. Y. Hou and X.-H. Wu. A multiscale finite element method for elliptic problems in composite materials and porous media. J. Comput. Phys., 134(1):169 - 189, 1997.

[16] T. J. R. Hughes, G. R. Feijóo, L. Mazzei, and J.-B. Quincy. The variational multiscale method-a paradigm for computational mechanics. Comput. Methods Appl. Mech. Engrg., 166(1-2):3-24, 1998.

[17] T. J. R. Hughes and G. Sangalli. Variational multiscale analysis: the fine-scale green's function, projection, optimization, localization, and stabilized methods. SIAM J. Numer. Anal., 45(2):539557, 2007.

[18] A. Målqvist and D. Peterseim. Localization of elliptic multiscale problems. Math. Comp., $83(290): 2583-2603,2014$.

[19] M. Ohlberger and B. Verfürth. Analysis of multiscale methods for the two-dimensional Helmholtz equation with highly heterogeneous coefficient. Part II. Two-scale localized orthogonal decomposition. arXiv:1605.03410, 2016. 
[20] H. Owhadi and L. Zhang. Localized bases for finite-dimensional homogenization approximations with nonseparated scales and high contrast. Multiscale Model. Simul., 9(4):1373-1398, 2011.

[21] H. Owhadi, L. Zhang, and L. Berlyand. Polyharmonic homogenization, rough polyharmonic splines and sparse super-localization. ESAIM Math. Model. Numer. Anal., 48(2):517-552, 2014.

[22] D. Peterseim. Variational multiscale stabilization and the exponential decay of fine-scale correctors. In G. R. Barrenechea, F. Brezzi, A. Cangiani, and E. H. Georgoulis, editors, Building Bridges: Connections and Challenges in Modern Approaches to Numerical Partial Differential Equations, Lecture Notes in Computational Science and Engineering. Springer, May 2016.

[23] D. Peterseim and R. Scheichl. Robust numerical upscaling of elliptic multiscale problems at high contrast. Computational Methods in Applied Mathematics, 2016.

[24] R. Scheichl, P. S. Vassilevski, and L. T. Zikatanov. Multilevel methods for elliptic problems with highly varying coefficients on nonaligned coarse grids. SIAM J. Numer. Anal., 50(3):1675-1694, 2012 .

[25] L. R. Scott and S. Zhang. Finite element interpolation of nonsmooth functions satisfying boundary conditions. Math. Comp., 54(190):483-493, 1990.

[26] R. Verfürth. A note on polynomial approximation in Sobolev spaces. M2AN: Math. Model. Numer. Anal., pages 715-719, 1999. 